\title{
Single trajectory transport coefficients and the energy landscape by Molecular Dynamics Simulations
}

\author{
D.M. Heyes ${ }^{* \dagger}$ and D. Dini ${ }^{\ddagger}$ \\ Department of Mechanical Engineering, \\ Imperial College London, \\ Exhibition Road, South Kensington, \\ London SW7 2AZ, United Kingdom. \\ E.R. $\operatorname{Smith}^{\S}$ \\ Department of Mechanical and Aerospace Engineering, \\ Brunel University London, Uxbridge, \\ Middlesex UB8 3PH, United Kingdom. ๆ
}

(Dated: April 18, 2020)

* Author to whom correspondence should be addressed: d.heyes@imperial.ac.uk 


\begin{abstract}
The Green-Kubo (GK) method is widely used to calculate the transport coefficients of model liquids by Molecular Dynamics (MD) simulation. A reformulation of GK was proposed in [D.M. Heyes, E.R. Smith and D. Dini J. Chem. Phys., 150, 174504 (2019)] which expressed the shear viscosity in terms of a probability distribution function (PDF) of 'single trajectory viscosities' (ST), called 'viscuits'. This approach is extended here to the bulk viscosity, thermal conductivity and diffusion coefficient. The PDFs of the four ST expressed in terms of their standard deviations (calculated separately for the positive and negative sides) are shown by MD to be statistically the same. This PDF can be represented well by a sum of exponentials, and is independent of system size and state point in the equilibrium fluid regime. The PDF is not well reproduced by a stochastic model. The PDF is statistically the same as that derived from the potential energy, $u$, and other thermodynamic quantities, indicating that the transport coefficients are determined quantitatively by and follow closely the time evolution of the underlying energy landscape. The PDFs of out-of-equilibrium supercooled high density states are quite different to those of the equilibrium states.
\end{abstract}




\section{INTRODUCTION}

The rough potential energy surface or landscape (PEL) on which molecules in a liquid move is composed of a wide range of near-harmonic valleys about which the molecules oscillate separated by saddle points which allow for intervalley transitions involving local configurational changes. This has been a longstanding interest in the role played by the PEL on viscous and other transport coefficients. ${ }^{1}$ A number of phenomenological theories of self-diffusion and shear viscosity of the liquid state are based on certain assumptions about the underlying PEL and its time dependent evolution. These include, for example, Eyring's thermally and stress activated basin hopping shear thinning formula, ${ }^{2}$ and Zwanzig's model of self-diffusion in which the liquid is viewed to consist of regions of phase space in which the configuration oscillates before it diffuses into another 'cell' through a saddle point. ${ }^{3,4}$ The related shoving, ${ }^{5,6}$ and asymmetry, ${ }^{7}$ models assume that the activation energy for a flow event is supplied by the reorganizational release of the shear elastic energy arising from deformation of a group of molecules by an applied shear field. More specific descriptions of the evolution of the system through its energy landscape are provided by Stillinger's inherent structures approach, ${ }^{8}$ involving a quench to the nearest PEL basin, which has been used to describe supercooled liquids and glassy systems, ${ }^{9,10}$. The Instantaneous Normal Modes (INM) approach. ${ }^{11-15}$ without quenching characterizes the states explored at the given temperature. These latter theories are based on the assumption that liquid molecular dynamics can be formulated in terms of collective variables analogous to the phonons used to describe solids. Molecular Dynamics (MD) computer simulation has played an invaluable role in testing these approximate theories. The Soft Glassy Rheology or SGR trap model of soft materials ${ }^{16-18}$ builds on these mean field liquid state models on taking the zero temperature limit. 
The connections between the potential energy landscape and its time dependence, and the processes that lead to the values of the transport coefficients of liquids are still not well established at a qualitative and quantitative level. MD is suited to investigate these connections, which is the subject of this work.

There are two formally equivalent equilibrium MD methods for determining the transport coefficients (TC) of model liquids. These are the Green-Kubo (GK) time correlation function approach and the Einstein-Kubo-Helfand (EKH) equations. ${ }^{19-22}$ Another, 'hybrid' route which shares features of both approaches was discussed by Erpenbeck (see Eq. (11) in Ref. 19). This third method has not been extensively employed in the literature, but as will be seen in Sec. II.A has particular relevance to this work's methodology. The hybrid method can be rewritten in terms of 'single trajectory' (ST) 'events' whose combined effect gives the transport coefficient. Stillinger and Debenedetti pioneered this approach to analyse in more detail the dynamical processes underpinning the self-diffusion coefficient and shear viscosity. ${ }^{23,24}$ The application of this approach in this work is focused on the transport coefficients themselves. This formulation was initiated for the shear viscosity, $\eta_{s}$, in Ref. 25, in which the ST event was referred to as a 'viscuit', $\eta_{s, u}$ to emphasize its role as a building block, which when summed gives the viscosity. The shear viscosity is the first moment of the viscuit PDF. Unlike the viscosity itself, $\eta_{s, u}$, can be negative which indicates entropy decrease during these trajectories on the application of a small shear rate (see pages 297-299 in Ref. $^{26}$ ). The PDF of $\eta_{s, u}$ for long times has a large negative stress region, but its first moment is positive as it must be to give a positive viscosity for the system. ${ }^{25}$

The advantage of the ST approach is that the viscuit is a fundamental quantity which provides additional information on the dynamical events that when added up give the 
magnitude of the transport coefficient. In this work the ST PDF analysis is extended to the bulk viscosity, thermal conductivity and self-diffusion for a range of equilibrium and out-of-equilibrium Lennard-Jones (LJ) state points. The analytic form of the PDFs and their sensitivity to system parameters are examined. The relationship between the transport coefficient PDFs and those characterizing the potential energy landscape are also explored.

In Sec. II.A the key equations of the ST approach are derived from the Green-Kubo method (GK) for the shear viscosity. Extensions to other transport coefficients are made in Sec. II.B. Computational details of the MD simulations are given in Sec. II.C and simulation results of the ST properties are presented in Sec. III. A summary of the conclusions of this work is made in Sec. IV. 


\section{THEORY AND COMPUTATIONAL DETAILS}

In this section the equations used to compute the ST-related quantities and computational details are given.

\section{A. Shear viscosity}

The Green-Kubo (GK) method for calculating the shear viscosity is, ${ }^{26}$

$$
\eta_{s, G K}(t)=\frac{V}{k_{B} T} \int_{0}^{t}<P_{y z}(0) P_{y z}(x)>d x, \quad \eta_{s}=\lim _{t \rightarrow \infty} \eta_{s, G K}(t),
$$

where $<P_{y z}(0) P_{y z}(x)>$ is the shear stress autocorrelation function, and the time origin is defined to be at $x=0$. The shear viscosity, $\eta_{s}$, is determined in Eq. (1) from one of the off-diagonal elements of the pressure tensor, and the element, $P_{y z}$ is chosen for clarity. For a monatomic fluid,

$$
P_{y z}=\frac{1}{V}\left(\sum_{i=1}^{N}\left[m_{i} v_{y i} v_{z i}-\frac{1}{2} \sum_{j \neq i}^{N} r_{y, i j} \frac{r_{z, i j}}{r_{i j}} \phi^{\prime}\left(r_{i j}\right)\right]\right),
$$

where $N$ is the number of molecules in volume $V$ (i.e., the volume of the simulation cell here), $v_{\alpha i}$ is the $\alpha$ component of the velocity of molecule, $i$, or $\boldsymbol{v}_{i}$, and $r_{\alpha, i j}$ is the $\alpha$ component of the pair separation vector between molecules $i$ and $j$. The first derivative of the pair potential, $\phi(r)$, with respect to $r$ is denoted by $\phi^{\prime}$. The shear viscosity, $\eta_{s}$, is the large $t$ limit of the 'time dependent viscosity', $\eta_{s}(t)$ as defined in Eq. (1).

The GK formula in Eq. (1) can be transformed into the ST form by reversing the order of the integral and the time origin average, $\langle\cdots\rangle$, in Eq. (2),

$$
\begin{aligned}
\eta_{s, S T}(t) & =<\frac{V}{k_{B} T} \int_{0}^{t} P_{y z}(0) P_{y z}(x) d x>, \\
& =<\eta_{s, u}(t)>\text {, where } \\
\eta_{s, u}(t) & =\frac{V}{k_{B} T} \int_{0}^{t} P_{y z}(0) P_{y z}(x) d x
\end{aligned}
$$




$$
\begin{aligned}
& =\frac{V}{k_{B} T} P_{y z}(0) \int_{0}^{t} P_{y z}(x) d x \\
\eta_{s, S T}(t) & =\int_{-\infty}^{\infty} d \eta_{s, u}(t) \eta_{s, u}(t) P_{\eta s}\left(\eta_{s, u}(t)\right), \\
\eta_{s} & =\lim _{t \rightarrow \infty} \eta_{s, S T}(t),
\end{aligned}
$$

where $P_{\eta s}\left(\eta_{s, u}(t)\right)$ is the probability distribution function of the single trajectory viscosity or $\mathrm{ST}, \eta_{s, u}(t)$. In the penultimate line of Eq. (3) the time dependent viscosity is written as the first moment of the viscuit PDF. The $\eta_{s, u}(t)$ involves an integral up to time $t$, and is defined starting from the value of the pressure tensor at a single specific time ('time origin') during the simulation i.e., $P_{y z}(0)$. There is a different PDF for each value of $t$, but the PDF will converge to a limiting form for large $t$. In practice the influence of the intital stress dies away rather quickly for liquids at equilibrium, typically lasting only for a few Maxwell relaxation times, $\tau_{M}=\eta_{s} / G_{\infty}$. The quantities defined in Eq. (3) can be obtained from equilibrium MD simulations. The $\eta_{s, u}(t)$ is basically the hybrid method, ${ }^{19}$ applied on an individual trajectory basis. This computation adds little to the simulation computational time as the viscuits are already calculated in the GK procedure, so both approaches can be carried out simultaneously.

\section{B. Other transport coefficients}

The same reformulation of the GK formulas can be made for the other transport coefficients.

\section{Bulk viscosity}

The analogous treatment for the bulk viscosity, ${ }^{27-30}$ is as follows. The instantaneous value of the pressure, $P(t)$, is

$$
P=\frac{1}{3 V}\left(\sum_{i=1}^{N}\left[m_{i} v_{i}^{2}-\frac{1}{2} \sum_{j \neq i}^{N} r_{i j} \phi^{\prime}\left(r_{i j}\right)\right]\right)
$$


using the virial formula. The deviatoric pressure in a constant $N$ ensemble is, ${ }^{28}$

$$
\delta P(t)=P(t)-\bar{P}-\frac{1}{V}\left\{\left(\frac{\partial \bar{P}}{\partial e}\right)_{\rho}[E(t)-\bar{E}]\right\} .
$$

where $e$ is the internal energy per unit volume. ${ }^{30}$ The time averages of the total energy and pressure are $\bar{E}$ and $\bar{P}$, respectively. The simulations of the bulk viscosity need to be carried out in the NVE ensemble for the final term in Eq. (5) to be zero.

The corresponding expressions to those in Eq. (3) for the bulk viscosity and corresponding ST are ${ }^{28-32}$

$$
\begin{aligned}
\eta_{b, G K}(t) & =\frac{V}{k_{B} T} \int_{0}^{t}<\delta P(0) \delta P(x)>d x, \quad \eta_{b, G K}=\lim _{t \rightarrow \infty} \eta_{b, G K}(t), \\
\eta_{b, S T}(t) & =<\frac{V}{k_{B} T} \delta P(0) \int_{0}^{t} \delta P(x) d x> \\
& =<\eta_{b, u}(t)>, \text { where } \quad \eta_{b, u}=\frac{V}{k_{B} T} \delta P(0) \int_{0}^{t} \delta P(x) d x \\
\eta_{b, S T}(t) & =\int_{-\infty}^{\infty} d \eta_{b, u}(t) \eta_{b, u}(t) P_{\eta b}\left(\eta_{b, u}(t)\right), \quad \eta_{b, S T}=\lim _{t \rightarrow \infty} \eta_{b, S T}(t),
\end{aligned}
$$

The function $P_{\eta b}$ is the PDF for the bulk viscosity which corresponds to $P_{\eta s}$ which is given in Eq. (3) for the shear viscosity.

\section{Thermal Conductivity}

The thermal conductivity, $\lambda$, involves the heat flux vector, $\boldsymbol{J}_{q}{ }^{33}$

$$
\boldsymbol{J}_{q}=\frac{1}{V}\left(\sum_{i=1}^{N}\left[e_{i} \boldsymbol{v}_{i}-\frac{1}{2} \sum_{j \neq i}^{N}\left(\boldsymbol{r}_{i j} \cdot \boldsymbol{v}_{i j}\right) \frac{\boldsymbol{r}_{i j}}{r_{i j}} \phi^{\prime}\left(r_{i j}\right)\right]\right),
$$

where

$$
e_{i}=\frac{1}{2} m_{i} v_{i}^{2}+\frac{1}{2} \sum_{j \neq i} \phi\left(r_{i j}\right)
$$

is the energy of a molecule in the fluid, $\boldsymbol{v}_{i j}=\boldsymbol{v}_{i}-\boldsymbol{v}_{j}$ is the relative velocity between molecules $i$ and $j$. The thermal conductivity, $\lambda$, and related quantities are, ${ }^{33,34}$,

$$
\lambda_{G K}(t)=\frac{V}{3 k_{B} T^{2}} \int_{0}^{t}<\boldsymbol{J}_{q}(0) \cdot \boldsymbol{J}_{q}(x)>d x, \quad \lambda_{G K}=\lim _{t \rightarrow \infty} \lambda_{G K}(t),
$$




$$
\begin{aligned}
\lambda_{S T}(t) & =<\frac{V}{3 k_{B} T^{2}} \boldsymbol{J}_{q}(0) \cdot \int_{0}^{t} \boldsymbol{J}_{q}(x) d x>, \\
& =<\lambda_{u}(t)>, \text { where } \quad \lambda_{u}=\frac{V}{3 k_{B} T^{2}} \boldsymbol{J}_{q}(0) \cdot \int_{0}^{t} \boldsymbol{J}_{q}(x) d x \\
\lambda_{S T}(t) & =\int_{-\infty}^{\infty} d \lambda_{u}(t) \lambda_{u}(t) P_{\lambda}\left(\lambda_{u}(t)\right), \quad \lambda_{S T}=\lim _{t \rightarrow \infty} \lambda_{u}(t),
\end{aligned}
$$

where $P_{\lambda}$ is the PDF for the thermal conductivity ST.

\section{Self-diffusion}

The fourth transport coefficient considered here is the self-diffusion coefficient, $D$, which is a single particle property. This can be calculated using a Green-Kubo (GK) formula, ${ }^{26}$ employing the velocity autocorrelation function (VACF),

$$
\begin{aligned}
D_{G K}(t) & =\frac{1}{3} \int_{0}^{t}<\boldsymbol{v}(0) \cdot \boldsymbol{v}(x)>d x, \quad D_{G K}=\lim _{t \rightarrow \infty} D_{G K}(t), \\
D_{S T}(t) & =<\frac{1}{3} \boldsymbol{v}(0) \cdot \int_{0}^{t} \boldsymbol{v}(x) d x>, \\
& =<D_{u}(t)>, \text { where } \quad D_{u}=\frac{1}{3} \boldsymbol{v}(0) \cdot \int_{0}^{t} \boldsymbol{v}(x) d x \\
D_{S T}(t) & =\int_{-\infty}^{\infty} d D_{u}(t) D_{u}(t) P_{D}\left(D_{u}(t)\right), \quad D_{S T}=\lim _{t \rightarrow \infty} D_{u}(t),
\end{aligned}
$$

where $\boldsymbol{v}(t)$ is the velocity of an arbitrary molecule at time $t$, and $P_{D}$ is the PDF of the self-diffusion ST.

Although strictly speaking the term 'viscuit' was introduced for the shear viscosity in Ref. 25, it will be used in this work as a general term for the other ST transport (and thermodynamic) quantities and their PDFs .

\section{Probabiity distribution function, PDF}

Various definitions of the viscuit PDFs will be considered in this section.

\section{PDFs with the two sides normalized separately}

It was shown in Ref. 25 that in LJ units the PDF on the positive and negative ST sides are not 
symmetric about $\eta_{s, u}=0$, but skewed to the positive side as the viscosity must be positive. As the two sides of the PDF are different it is convenient to treat the two sides separately and to establish relationships between them. The properties of the total PDF are obtained by combining those of its two sides, in a suitably weighted manner. For generality, let $X$ denote one of the four transport coefficients discussed in Secs. II.A and II.B, and $X_{u}$ be the corresponding ST quantity. Each side of the total PDF is normalized to unity, which means that the form it takes depends on the units used for the ST argument.

Let $x_{-}=X_{u} / \sigma_{-}$, where $X_{u}<0$ and $\sigma_{-}$is the standard deviation of $X_{u}$ in the same units on the negative side. The quantity $x_{-}$is dimensionless. Also let $N\left(x_{-} \pm \Delta x_{-} / 2\right)$ be the number of viscuits in the range $x_{-} \pm \Delta x_{-} / 2$ for a given sample length. Then the relevant PDF of the viscuits on the negative side is, $P_{-}\left(x_{-}\right)$,

$$
P_{-}\left(x_{-}\right)=\lim _{\Delta x_{-} \rightarrow 0} \frac{1}{N_{-}}\left[\frac{\Delta N\left(x_{-} \pm \frac{\Delta x_{-}}{2}\right)}{\Delta x_{-}}\right]
$$

where $N_{-}$is the number of ST on the negative side. The corresponding PDF for the positive side is $P_{+}\left(x_{+}\right)$is also defined by Eq. (11) with the subscript '-' replaced by ' + ' throughout. This notation convention is used generally for other quantities here. As will be demonstrated, this PDF definition is perhaps the most fundamental of all of the types considered in this work because the argument is independent of the units of $X_{u}$. Let $\bar{X}$ be the sample average of the $X_{u}$ combined for both sides of the distribution and $\left\langle X_{u,+}\right\rangle$ be the sample average of the positive ST. These quantities are formally related via,

$$
\begin{aligned}
\bar{X} & =R_{+}<X_{u,+}>+R_{-}<X_{u,-}>\quad \text { where }, \\
<X_{u,+}> & =\int_{+} X_{u} P_{+}(x) d x=\sigma_{+} \int_{+} x P_{+}(x) d x \\
& \equiv \sigma_{+} I_{+} \quad \text { and } \quad I_{+}=\int_{+} x P_{+}(x) d x \quad x>0, \\
<X_{u,-}> & \equiv-\sigma_{-} I_{-} \quad \text { and } I_{-}=-\int_{-} x P_{-}(x) d x \quad x<0,
\end{aligned}
$$




$$
\bar{X}=R_{+} \sigma_{+} I_{+}-R_{-} \sigma_{-} I_{-}
$$

where $\int_{+}$denotes integration over the positive side, $R_{+}$is the fraction of the total $X_{u}$ events that are positive and $x$ represents either $x_{-}=X_{u} / \sigma_{-}$or $x_{+}=X_{u} / \sigma_{+}$depending on which side of the PDF is being considered. Note that $I_{+}$and $I_{-}$are both defined as positive quantities, and $R_{-}=1-R_{+}$. The standard deviations $\sigma_{-}$and $\sigma_{+}$have the same units as $\bar{X}$.

In order to make further analytic progress it is necessary to consider the relationship between $R_{-}$ and $R_{+}$, and $I_{-}$and $I_{+}$. There is no obvious proof or derivation which establishes these relations. It will be shown in the results section that to a good approximation, $P_{+}(x)=P_{-}(-x) \equiv P(x)$, and therefore $I_{+}=I_{-} \equiv I$. However, $R_{-}$cannot be determined by rigorous derivation, at least as far as we are aware. The formally exact result is,

$$
\begin{aligned}
& R_{+}=\frac{N_{+}}{N_{-}+N_{+}}=\frac{R_{N}}{1+R_{N}} \\
& R_{-}=\frac{N_{-}}{N_{-}+N_{+}}=\frac{1}{1+R_{N}} .
\end{aligned}
$$

where $N_{+}$is the total number of viscuits on the positive side of the distribution. It is reasonable to assume that $R_{N}=N_{+} / N_{-} \geq 1$ but this would need to be demonstrated in practice. Combining Eqs. (12) and (13) leads to,

$$
\begin{aligned}
\bar{X} & =R_{+} \sigma_{+} I_{+}-R_{-} \sigma_{-} I_{-}, \\
& =\frac{R_{N} \sigma_{+} I_{+}-\sigma_{-} I_{-}}{1+R_{N}}, \\
& =\frac{R_{N} \sigma_{+}-\sigma_{-}}{1+R_{N}} I \text { if } I_{+}=I_{-} \equiv I, \\
I \equiv I_{N} & =\left[\frac{1+R_{N}}{R_{N} \sigma_{+}-\sigma_{-}}\right] \bar{X}
\end{aligned}
$$

for each transport coefficient. The advantage of this prescription for $R_{+}$and $R_{-}$is that the ratio $N_{+} / N_{-}, \sigma_{+}$and $\sigma_{-}$can be obtained directly and independently from an MD simulation. The last two lines in Eq. (14) follow from the assumption that, $I_{+}=I_{-} \equiv I$. Equation (14) 
shows that $\bar{X}=0$ if $R_{N}=1, \sigma_{+}=\sigma_{-}$and $I_{+}=I_{-}$.

Alternatively we may go further and propose that, for example,

$$
\begin{aligned}
& R_{+}=\frac{\sigma_{+}}{\sigma_{-}+\sigma_{+}}=\frac{R_{\sigma}}{1+R_{\sigma}}, \\
& R_{-}=\frac{\sigma_{-}}{\sigma_{-}+\sigma_{+}}=\frac{1}{1+R_{\sigma}}=1-R_{+},
\end{aligned}
$$

where $R_{\sigma}=\sigma_{+} / \sigma_{-} \geq 1$, and therefore

$$
\begin{aligned}
\bar{X} & =R_{+} \sigma_{+} I_{+}-R_{-} \sigma_{-} I_{-}, \\
& =\frac{\sigma_{+}^{2}}{\sigma_{-}+\sigma_{+}} I_{+}-\frac{\sigma_{-}^{2}}{\sigma_{-}+\sigma_{+}} I_{-}, \\
& =\frac{\sigma_{+}^{2}-\sigma_{-}^{2}}{\sigma_{\bar{R}+\sigma_{+}}} I, \quad \text { if } \quad I=I_{+}=-I_{-} \\
& =\left[\frac{R_{\sigma} \sigma_{+}-\sigma_{-}}{1+R_{\sigma}}\right] I \\
I \equiv I_{\sigma} & =\left[\frac{\sigma_{+}+\sigma_{-}}{\sigma_{+}^{2}-\sigma_{-}^{2}}\right] \bar{X} \quad \text { where } \quad \sigma_{+}>\sigma_{-}, \\
& =\left[\frac{R_{\sigma}+1}{R_{\sigma} \sigma_{+}-\sigma_{-}}\right] \bar{X} .
\end{aligned}
$$

\section{PDF with dimensioned arguments}

PDFs where the argument is in different units can also be derived straightforwardly from $P_{-}$ and $P_{+}$. For example, the PDF where the $\mathrm{ST}$ is in 'real' (i.e., here, LJ ) units is,

$$
\begin{aligned}
P_{L J}\left(X_{u}\right) & =\lim _{\Delta X_{u} \rightarrow 0}\left[\frac{\Delta N\left(X_{u} \pm \frac{\Delta X_{u}}{2}\right)}{\Delta X_{u}}\right], \\
& =\lim _{\Delta x \rightarrow 0}\left[\frac{\Delta N\left(X_{u} \pm \frac{\Delta X_{u}}{2}\right)}{\Delta x \sigma_{d}}\right] \\
& =\frac{P(x)}{\sigma_{d}}
\end{aligned}
$$

where the PDFs are for each side of the distribution taken separately. The standard deviation, $\sigma_{d} \equiv \sigma_{-}$or $\sigma_{-}$, and $\Delta X_{u}=\Delta x \sigma_{d}$, are all in LJ units. Note that $\Delta N\left(X_{u} \pm \frac{\Delta X_{u}}{2}\right)=\Delta N\left(x_{u} \pm\right.$ $\frac{\Delta x_{u}}{2}$. Another possible form for the ST is to express it in terms of the transport coefficient, $X$, itself, which can be obtained independently using the GK method, for example.

$$
P_{X}\left(\frac{X_{u}}{X}\right)=\lim _{\Delta X_{u} / X \rightarrow 0}\left[\frac{\Delta N\left(\frac{X_{u}}{X} \pm \frac{\Delta X_{u}}{2 X}\right)}{\Delta \frac{X_{u}}{X}}\right],
$$




$$
\begin{aligned}
& =X \lim _{\Delta X_{u} / X \rightarrow 0}\left[\frac{\Delta N\left(\frac{X_{u}}{X} \pm \frac{\Delta X_{u}}{2 X}\right)}{\Delta X_{u}}\right], \\
& =X \lim _{\Delta X_{u} / X \rightarrow 0}\left[\frac{\Delta N\left(\frac{X_{u}}{X} \pm \frac{\Delta X_{u}}{2 X}\right)}{\Delta x \sigma_{d}}\right], \\
& =\frac{X}{\sigma_{d}} P(x)
\end{aligned}
$$

The PDFs presented in the Results section III.B were computed from the MD simulations to a resolution of typically, $x \simeq 0.008$ standard deviation units, and for a ST range of at least $c a$. \pm 8 standard deviations on both sides of the distribution.

To summarize, the transport coefficient can be obtained by the Green-Kubo or PDF routes given in Eq. (3) for the shear viscosity, Eq. (5) for the bulk viscosity Eq. (9) for the thermal conductivity and Eq. (10) for the self-diffusion. A PDF method for obtaining these transport coefficients is also given in these equations. In Ref. 25 it was shown that the viscuit PDF was asymmetric about the ordinate axis. In order to make further theoretical progress, the PDFs $P_{ \pm}$defined above were introduced which are calculated and normalized separately for the two sides. The difference between these PDFs, and $P_{L J}$ and $P_{X}$ is that the latter (from their initial definitions) are defined and normalized by the same quantity over the whole viscuit domain (i.e., not the two halves separately).

A more direct route to obtain the transport coefficient is to simply add the individual viscuits as they occur in the simulation. Written as a summation over the ST negative and positive argument contributions, this is

$$
\bar{X}=\frac{\sum_{+-} X_{u}}{N_{-}+N_{+}}=\frac{\sum_{-} X_{u}}{N_{-}+N_{+}}+\frac{\sum_{+} X_{u}}{N_{-}+N_{+}}
$$

where $\sum_{+-}, \sum_{-}$and $\sum_{+}$indicate the sum over all, the negative and positive ST, respectively. Equation (19) is referred to as the 'direct' viscuit method to obtain the transport coefficient. 


\section{Simulation Details}

The simulations were carried out using the Lennard-Jones (LJ) pair potential, $\phi_{L J}(r)=4 \epsilon\left[(\sigma / r)^{12}-(\sigma / r)^{6}\right]$, where $\epsilon$ and $\sigma$ define the characteristic energy of interaction and diameter of the molecule, respectively. The results presented are in LJ units of $\epsilon, \sigma$ and $m$, the mass of each molecule. The MD time step was, $\Delta t=0.004 / \sqrt{T}$, and the interaction truncation distance, $r_{c}$ was 2.5 (see Ref. 35). The numbers of particles in the simulation cell, $N$, were $500,864,2048$ and 4000 to assess the system size dependence of the results (most of the simulations and figures presented employed $N=864$ ). The computations were conducted typically for $2 \times 10^{7}$ time steps for $N=864$ and $10^{6}$ for $N=2048$ for each state point during the post-equilibration stage. Four representative fluid state points specified in Table I were used in the simulations. State points A-C are in the equilibrium fluid part of the phase diagram while state point D is a supercooled (metastable) liquid. The LJ state point $\mathrm{C}$ in Table I, $\rho=0.8442$ and $T=0.722$ was introduced by Levesque, Verlet and coworkers, ${ }^{37,38}$ and has been used since as a standard reference state point in many studies. State point $\mathrm{C}$, which is quite close to the triple point, was used as the default state point to produce most of the figures (unless stated otherwise). Simulations were carried out using NVE and NVT dynamics, where in the latter case the Nosé-Hoover thermostat was used, ${ }^{39}$ with a time constant of 3 LJ time units. Statistical uncertainties in the mean values were determined using block averaging. ${ }^{40}$ Four transport coefficients were considered, whose average values for the four state points are given in Table 1. 


\section{RESULTS AND DISCUSSION}

In this section the PDFs for the four transport coefficients are compared. In some examples, the statistical characteristics of the PDFs on the negative and positive sides were determined separately, as were their contributions to the total value of the transport coefficient. In other cases the PDF was calculated by treating the negative and positive regions as a single domain and the PDF normalized as a single entity.

\section{A. Probability distribution functions and the transport coefficients}

Figure 1(a) presents the Green-Kubo time correlation functions used to compute by time integration the four transport coefficients for $\rho=0.8442$ and $T=0.722$ (state point ' $\mathrm{C}$ ' in Table I). The correlation functions are normalized to unity at $t=0$ for ready comparison. Figure 1(b) shows the corresponding time dependent transport coefficients, $X(t)$ defined in Eqs. (3), (6), (9), and (10), for $\eta_{s}, \eta_{b}, \lambda$ and $D$ respectively. At this state point a plateau is reached in $X(t)$ by $c a$. $t \simeq 2.5$ for all four transport coefficients. The PDFs depend on the time, $t$ of the ST integral. This time is long enough for the time integral of the correlation function to reach a plateau and give a reliable value for the shear viscosity by the GK method. This is also the time required to obtain long time limiting forms for the PDFs.

Figure 2 presents the viscuit probability distributions for $t=2.5$ where the viscuit is in LJ units (in frame (a)) i.e., $P_{L J}$ from Eq. (17). Frame (b) shows $P_{X}$ defined in Eq. (18) where the viscuit is normalized by the average shear viscosity. The PDF normalization was computed over the combined negative and positive sides of the distribution (i.e., treating it as a single domain). Both types of PDF are asymmetric, with the PDF ultimately decaying more slowly on the positive side, which is evident in the difference curves also shown on the figure. This 
is necessary for the viscosity (the first moment of the PDF) to be positive. One could view the positive viscuits as being the entropy producing trajectories when a small shear field is applied. The negative viscuits are the corresponding entropy 'absorbing' trajectories. In Zwanzig's theory of self-diffusion these would be associated with the liquid-like (diffusive or heat producing dissipative) and solid-like (oscillatory) events, respectively, ${ }^{3,4}$ although with collective property transport coefficients such as viscosity and thermal conductivity the spatial aspects of these processes are not so easy to picture. The PDF has a cusp in the small viscuit limit on both sides, which is more peaked and short ranged on the negative viscuit side.

Figure 3 shows the accumulated first moment of the $X_{u}$ PDF, $P_{L J},(t=2.5)$ for the four transport coefficients in LJ units obtained by the MD simulations evaluated at state point C. The plateau values on the right of the figure are the values of the transport coefficients. The argument of the PDF is in units of the standard deviation $\sigma_{d}$ of $X_{u}$ for each side. The figure reveals that the limiting value (indicated by the GK values) is only achieved with $X_{u}$ after about 8 standard deviations. Therefore although the viscuit events are quite infrequent in that part of the PDF (evidenced by its small value) their magnitude is such that they contribute a non-neglible amount to the transport coefficient. The GK values for these transport coefficients are indicated by the horizontal lines on the figure, revealing very good agreement in the large argument limits.

Figure 4 presents the time dependent transport coefficient obtained by integration of the PDFs and also by GK, both up to time $t$. More data of a similar kind for other quantities and state points is given in supplementary material. The figure also shows the contributions to the transport coefficients from the negative and positive sides of the PDF. Frame (a) is for the shear viscosity and frame (b) is for the self-diffusion coefficient. For large $t$ the contributions from the 
two sides are larger in magnitude than the summed or total value. They continue to increase in magnitude even when the total viscosity has reached a plateau. This trend is considered to be due to noise in the viscuit for long times (say, $t>2.5$ ). Positive and negative values for the viscosity (viscuit) will appear randomly with equal probability as time increases and the $\eta_{s,-}$ and $\eta_{s,+}$ will increase continuously with time without reaching a plateau. This is not a practical problem as this noise-related feature for practical purposes cancels out in the total transport coefficient. The peak in the total self-diffusion coefficient is associated with the cage effect around each molecule which produces a negative region in the velocity autocorrelation function.

\section{B. PDFs with dimensionless arguments}

In this section the ST PDFs whose argument is normalized by its standard deviation are considered. The PDF of each side of the distribution is treated separately. Figure 5 shows the $t=2.5 \mathrm{PDF}$ defined in Eq. (11) for $P_{-}$, and $P_{+}$for different numbers of particles in the simulation cell. The figure reveals that the PDF is statistically independent of $N$ and also the distribution is symmetrical about the ordinate axis, i.e., $P_{-}\left(\eta_{s, u}\right)=P_{+}\left(-\eta_{s, u}\right)$. In the bottom frame the $P_{ \pm}\left(\left|\eta_{s, u}\right|\right)$ for the two sides are plotted on a lin-log scale, which indicates that the wings on the two sides of the PDF for large ST values are exponential and coincident.

Figure 6 shows the $P_{ \pm}\left(\left|\eta_{s, u}\right| / \sigma_{d}\right)$ for the negative and positive sides of the distribution as a function of $t$. For a short integration time, $t=0.015$ and to a much lesser extent at $t=0.493$, there are differences between this definition of the PDF on the negative and positive sides for small $\left|\eta_{s, u}\right| / \sigma_{d}$ values. The agreement for large viscuit values is better for large $\left|\eta_{s, u}\right|$ even for small $t$. The two PDFs converge as $t$ increases to $t \simeq 2.5$, where the integral of the 
GK correlation function has reached a plateau value and is sufficient time to define the viscosity.

Figure 7 presents the $P_{ \pm}$for the shear viscosity ST on lin-lin and lin-log scales in frames (a) and (b), respectively, for state point C. Data for two (not too small) $t$ values are also presented, but are not distinguishable on the figure. The analytic formula,

$$
P\left(\left|\eta_{s, u}(t)\right| / \sigma_{d}\right)=\sum_{i=1}^{3} A_{i} \exp \left(-b_{i}\left|\eta_{s, u}(t)\right| / \sigma_{d}\right),
$$

where $A_{i}$ and $b_{i}$ are fit constants, matches the simulation data well. The constants in Eq. (20) are given in the caption to Fig. 7. Frame (a) shows that the slowest decaying exponential term captures the PDF for reduced ST greater than about 1.5 while the two other exponentials are also required to represent the PDF for about, $\left|\eta_{s, u}(t)\right| / \sigma_{d}<1.5$. More exponentials could be added to improve the agreement, but just three is sufficient for graphical purposes to demonstrate the adequacy of the general functional form in Eq. (20). PDFs with an exponential tail are frequently found in natural processes involving a wide spread of events of different magnitudes. The stronger events are associated with increasingly long waiting or renewal times. ${ }^{41}$

The representation of a time relaxation function by a stretched exponential or more generally a weighted sum of exponentials with a broad distribution of relaxation times is well established in many fields. ${ }^{42-45}$ Here the viscosity is framed in terms of a weighted sum of exponentials of the distribution of the ST (time is only implicitly present in this representation). The two constructions could be considered to be equivalent if each exponential term in time corresponds to one type of structural relaxation event characterized by the viscuit and its PDF (see Ref. 46 for further discussion). The rarer the event, which is equivalent to an escape from a stronger cage or deeper trap, the larger is the renewal or waiting time between their repeated 


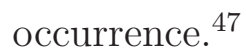

The state point dependence of the dimensionless PDF $P_{ \pm}$of the shear viscosity is shown in Fig. 8 for the four state points defined in Table I. These are given on lin-lin and lin-log scales in frames (a) and (b), respectively. The figure shows that the PDFs are all monotonically decaying and there is no statistically significant difference between them on the two sides for equilibrium state points A-C.

In contrast, the corresponding PDF of the out-of-equilibrium state (D) is highly oscillatory on the positive dissipative side indicative of a limited number of accessible relaxation paths in phase space, and similar to the equilibrium state point behavior on the negative side. The dynamical behavior of the 'events' of the system are quite different on the positive and negative sides of the PDF. Therefore these PDFs are a sensitive indicator of whether the state is at or out of equilibrium. The shear stress autocorrelation function (SACF) is monotonically and very slowly decaying, in contrast to the appearance of the viscuit PDF. This indicates that the viscuit PDF is providing additional information which is not visible in the SACF. As a qualifier it is worth noting the the GK method is not strictly valid for out-of-equilibrium state points like D because of the limited number of accessible states during a feasible simulation (see Ref. 40 p. 49). Further discussion of these differences is given in Sec. II.C.

The PDFs of the ST from the other transport coefficients are presented in Fig. 9 for state point C. The PDFs where the argument is normalized by the standard deviation are shown. The ST, $\eta_{b, u}, \lambda_{u}$ and $D_{u}$ used in their evaluation are defined in Eq. (6) for the bulk viscosity, Eq. (9) for the thermal conductivity, and Eq. (10) for self-diffusion, respectively. Figure 9(a) presents the reduced ST PDF for $t=0.49$ and $t=2.5$ for all four transport coefficients. The 
NVE ensemble is used in all four cases as the bulk viscosity defined in Eq. (6) is more readily computed in that ensemble (i.e., so that the $E(t)-\bar{E}$ term in Eq. (5) is zero). Frames (a) and (b) are in lin-lin and lin-log scales, respectively. Figure 9 reveals that the PDFs for the different transport coefficients are statistically indistinguishable.

To summarize, the standard deviation normalized PDF of the single trajectory transport coefficient is symmetric on its negative and positive sides. It is statistically independent of system size, state point, and choice of transport coefficient. The function can be represented very well by the sum of three exponentials, providing the state is a fluid in the equilibrium part of the phase diagram. This rather surprising result suggests that the ST PDF $P_{ \pm}$may be a function of fundamental statistical mechanical significance for transport coefficients. The PDF $P_{ \pm}$is symmetric on the two sides. The $P_{L J}$ and $P_{X}$ are asymmetric as is required for the transport coefficient to be positive, which is confirmed in Fig. 2. Suggestions for the origin and possible implications of these results are considered in Sec. III.C.

\section{PDF and transport coefficient statistical mechanics}

\section{Stochastic model}

The viscuit is the product of an instantaneous shear stress and the sum of its later values up to an arbitrary time difference, $t$. Each of these two quantities independently follows (normal) gaussian statistics because of the central limit theorem. The viscuit PDF (and its other transport coefficient analogues) can therefore be expressed as the product of two partially correlated quantities each individually exhibiting a gaussian PDF. The quantitative nature of this correlation is implicit in the MD results but lacks any separate theoretical description. It was shown in our previous publication, ${ }^{25}$ that PDFs generated by partially correlated random 
numbers using the Box-Muller numerical method gave poor agreement with the MD PDFs. It overestimated the asymmetry of the PDFs between the positive and negative viscuit sides of the distribution, which demonstrated the importance of viscuit longevity or persistence that apparently cannot be captured by a stochastic model.

There is an analytic stochastic model for the product of two partially correlated gaussian distributed quantities $G$ and $H$ both of zero mean. ${ }^{48-50,52}$ For transport coefficient, $U(t)$, let,

$$
\begin{aligned}
& U_{t} \equiv U(t)= G H_{t}, \quad \text { where for the shear viscosity, } \\
& G=\sqrt{A} P_{x y}(0) \quad H_{t}=\sqrt{A} \int_{0}^{t} P_{x y}(\alpha) d \alpha, \quad h_{t}=\frac{H_{t}}{t} \\
& \eta_{s}=<\lim _{t \rightarrow \infty} U(t)>.
\end{aligned}
$$

where $A=V / k_{B} T$ for the shear viscosity, and $P_{x y}$ in Eq. (21) would be replaced by the deviatoric pressure, heat flux and particle velocity for the other transport coefficients (see Eqs. (5), (9) and (10), respectively for the definitions of the $A$ parameter in each case). For the shear viscosity, for example, $\langle\cdots\rangle$ represents an average over the initial stress values, $P_{x y}(0)$. The PDF of $U_{t}$ is, ${ }^{48,49}$

$$
\begin{aligned}
P_{U}(u)= & B(c) D(c, u) E(c, u), \quad \text { where } \\
& B(c)=\frac{2}{\pi \sigma_{g} \sigma_{H t} \sqrt{1-c^{2}}}, \\
D(c, u) & =\exp \left(\frac{c u}{\sigma_{g} \sigma_{H t}\left(1-c^{2}\right)}\right), \\
E(c, u) & =K_{0}\left(\frac{|u|}{\sigma_{g} \sigma_{H t}\left(1-c^{2}\right)}\right) .
\end{aligned}
$$

The function, $K_{0}(x)=\int_{0}^{\infty} \exp (-x \cosh (\alpha)) d \alpha$, is the modified Bessel function of the second kind of zero order, which diverges in the $x \rightarrow 0$ limit. The quantities, $\sigma_{g}$ and $\sigma_{H t}$ are the standard deviations of $G$ and $H_{t}$, respectively, assuming $-\infty<u<\infty$ with zero mean. Because of the asymmetry of the viscuit PDF between the negative and positive sides when the 
viscuit is expressed in real units (not dimensionless), the MD generated standard deviations reported above are based on a half-normal or half-gaussian distribution which has a non-zero mean. The value of the half-gaussian standard deviation and mean contains the information specific to each transport coefficient and state point. The two definitions of the standard deviation are related through the multiplicative factor, $\sqrt{(\pi-2) / \pi}$ (the half-gaussian value being the smaller). The factor of 2 in the definition of $B(c)$ in Eq. (22) is to give the correct normalization for a half-gaussian based PDF. The quantity, $c$ in Eq. (21) denotes the correlation coefficient which is bounded by $-1<c<1$. For two quantities $x$ and $y$, with zero mean, $c$ is, $c=E(x y) / \sigma_{x} \sigma_{y}$, where $E(\cdots)$ is the expectation value, and $\sigma_{x}$ and $\sigma_{y}$ are the standard deviations of $x$ and $y$, respectively. In the present case $c$ is an unknown function of $G$ and $t$, and a mean-field theory value of $c$ is therefore used in Eq. (22) which is

$$
c=\frac{E\left(G h_{t}\right)}{\sigma_{g} \sigma_{h t}} \sim \sqrt{\frac{2 \tau_{c}}{t_{c}}}
$$

where $\tau_{c}$ is the relaxation time of the normalized time correlation function (for the shear viscosity, for example, $\left.\tau_{c}=\eta_{s} / G_{\infty}\right)$, and $t_{c}$ is the time at which the GK integral reaches a plateau value. The last equality in Eq. (23) follows from the EKH expression for the transport coefficient, ${ }^{20-22}$ which can be written as, $<h_{t}^{2}>=\left(2 \tau_{c} / t\right)<G^{2}>$. Typically $c$ is in the range 0.1 to 0.2 for the transport coefficients in the dense liquid state point regime considered here.

Figure 9(b) compares the MD-generated PDFs for the four transport coefficients with the predictions of Eq. (21) for three values of the correlation coefficient, $c$. The stochastic model PDF has a cusp for low ST, is singular at the origin and does not decay exponentially for large ST (it decays slower with increasing c). The MD-generated PDF also has a cusp near the origin but is probably finite at the origin owing the molecular discreteness of the system and decays exponentially for large arguments. The analytic stochastic model 
formula initially decays more rapidly than the MD PDF but crosses over to a smaller rate of decay in the large ST regime. It may be concluded that the deterministic nature of the $\mathrm{ST}$ is a crucial factor to include in any theoretical model of its PDF. As was discovered in Ref. 25, with a numerical stochastic model, the inclusion of a stochastic element distorts significantly the relative probability of occurrence of individual ST and is physically unrealistic.

\section{Ratio of the PDFs on the negative and positive sides}

Figure 10 shows the PDF ratios, $R=P_{L J,+} / P_{L J,-}$ for the same values of the absolute value of the ST (its negative and positive regions were combined and treated as a single domain for normalization purposes). The curves on the figures are for different integraton times, $t$, indicated on the figure. Frames (a) and (b) present $\ln (R)$ for the shear viscosity and thermal conductivity, respectively. The value of the time $t$ ranges from 0.05 to 0.49 which covers the initial decay period of the correlation function. The $\ln (R)$ data are linear with $\left|X_{u}\right|$ and the slope decreases with increasing $t$, The linearity of the dependence indicates that $R \propto \exp \left(A(t)\left|X_{u}\right|\right)$ for both transport coefficients, even at very short times. It was shown in Ref. 25 that $A(t)$ decays monotonically with $t$.

A simple model for $A(t)$ is derived here. Let the standard deviation, $\sigma_{d}$, of each side of the PDF be denoted by, $\sigma_{-}$or $\sigma_{+}$for the negative and positive sides, respectively. It follows from Eq. (17) that,

$$
R \equiv \frac{P_{L J,+}\left(X_{u,+}(L J)\right)}{P_{L J,-}\left(X_{u,-}(L J)\right)}=\frac{\sigma_{-}}{\sigma_{+}} \frac{P\left(X_{u,+} / \sigma_{+}\right)}{P\left(X_{u,-} / \sigma_{-}\right)}
$$

where $X_{u,+}, \sigma_{+}, X_{u,-}$ and $\sigma_{-}$are in the same units (i.e., LJ here). For $Y_{u, d} \equiv\left|X_{u}(L J)\right| / \sigma_{d}$ is greater than about 2, the PDF, $P$, decays to a good approximation as a single exponential, $P\left(X_{u}\right) \simeq C \exp \left(-\left|X_{u}\right| / \sigma_{d}\right)$, where the constant, $C$, is the same for both negative and positive 
sides (see Fig. 3). Therefore in this $Y_{u, d}$ region, taking the special case where, $X_{u}(L J)=$ $-X_{u,-}(L J)=X_{u,+}(L J)$,

$$
\begin{aligned}
R \equiv \frac{P_{L J,+}\left(X_{u,+}(L J)\right)}{P_{L J,-}\left(-X_{u,+}(L J)\right)} & \simeq \frac{\sigma_{-}}{\sigma_{+}} \exp \left[\left(\frac{1}{\sigma_{+}}-\frac{1}{\sigma_{-}}\right) X_{u}(L J)\right] \\
& =\frac{\sigma_{-}}{\sigma_{+}} e^{A X_{u}(L J)}
\end{aligned}
$$

where,

$$
A(t)=\frac{\sigma_{+}(t)-\sigma_{-}(t)}{\sigma_{+}(t) \sigma_{-}(t)} .
$$

For $Y_{u, d}<\sim 2$ the situation is more complicated as the PDF is not well represented by a single exponential. It was shown empirically in Ref. 25, Eq. (17), that $R$ has the general form as Eq. (25). Figure 11(a) compares the value of $A(t)$ obtained from the linear regression fits of Fig. 10 ('MD') with the predictions of Eq. (26). The analytic formula follows quite well the MD curve but slightly overestimates $A(t)$ particularly for large $t$.

In the approximate model of Eq. (26) the asymmetry of the two sides of the PDF (i.e., for here, $P_{L J}$ and $\left.P_{X}\right)$ is expressed in terms of the difference in the two standard deviations (or equivalently their ratio, $\sigma_{+} / \sigma_{-}$). Also the transport coefficient depends on the relative number of positive and negative ST, i.e., $R_{N}=N_{+} / N_{-}$, which occur in the simulation (see Eqs. (12) and (13)). There is no obvious formal relationship between these two ratios. It is more convenient to plot the inverse ratios as they are bounded by 0 and a number slightly less than 1. Figure 11(b) gives the dependence of, $\sigma_{-} / \sigma_{+}$against $N_{-} / N_{+}$for different times (implicit on the plot) and for the four transport coefficients obtained directly from the simulations. The figure shows that $N_{-} / N_{+} \propto \sigma_{-} / \sigma_{+}$for not too small $t$ values, as evident in the top right hand corner of Fig. 11(b). For short times (on the bottom left of the figure) it is evident that the relative number of negative ST decreases less rapidly than the 'range' of their PDFs as measured by the standard deviations. In fact for all values of $t$ the ratio 
$N_{-} / N_{+}>\sigma_{-} / \sigma_{+}$but they become closer in the large $t$ limit. Therefore, in general, there is not a trivial relationship between these two quantities, which characterize the two sides of the PDF. Note that the two ratios tend to zero in the bottom right hand corner as would be expected in the $t \rightarrow 0$ limit because closely spaced successive time step values are correlated.

The viscuit concept can be extended to any quantity of the system, $Y$, which is a function of the configurations

$$
\begin{aligned}
\delta Y(x) & =Y(x)-\bar{Y} \\
Y_{u}(t) & =\delta Y(0) \int_{0}^{t} \delta Y(x) d x
\end{aligned}
$$

where $\bar{Y}$ is the time average of $Y$. The average potential energy per particle, $u$, is an example of $Y$ which does not define a transport coefficient but is instead a representation of certain aspects of the potential energy landscape. The ratios, $N_{-} / N_{+}$and $\sigma_{-} / \sigma_{+}$can also be computed for this quantity. Figure 11(b) also shows that the $u$ data fall on the same curve for all $t$ as that of the transport coefficients. This indicates that the dynamical and statistical aspects of transport coefficients are coupled strongly to those of the underlying potential energy landscape of the system, which is intuitively what one would expect. There are studies of the configurational aspects of the potential energy landscape (i.e., the potential energy dependence of the number of saddle points ${ }^{51}$ ) but little on its time dependence. Further details of the computed ratios and other quantities for $t=2.5$ using state point $\mathrm{C}$ are given in Table III. A more complete list for all of the equilibrium state points for different times is given in supplementary material. Stillinger and Debenedetti also explored this hybrid approach in the context of the potential energy landscape, represented by inherent structure basins, to cast light on the Stokes-Einstein relation. ${ }^{23}$ 
Figure 12 presents a further analysis and comparison of the statistics of the viscuits and the potential energy landscape. The top frame shows a time trace of the local time averages for quantity $X$ (the shear stress or potential energy) and its associated ST. The time traces appear quite similar although the fluctuation features peaks and troughs do not coincide, which is presumably because the viscuit samples local anisotropies in the structure to a greater extent than the potential energy. Inspection of the time series indicates that the large outlier fluctuations are independent and random. The traces suggest that there is a distribution of energy minima with different depths usually closely bounded by energy maxima. The transits out of the minima are rapid and because of the neighboring maxima probably involve local rearrangement of the surrounding molecules as encapsulated in the shoving model..$^{5,6}$

The bottom frame of Fig. 12 shows the PDF of blocks of $N_{t}$ consecutive property values accumulated during the simulation, $X_{t}=\sum_{i=1}^{N_{t}} X_{i}$. The PDFs for shear stress (S) and potential energy, where the negative and positive sides of the distribution are treated separately, are given on the figure for very short and long times. The data collapse onto a gaussian (see the Fig. 12(b) caption for further details). This confirms that, independent of $N_{t}$, the property block summations exhibit a normal distribution in accordance with the central limit theorem. In contrast, the corresponding viscuit PDFs are exponential in character (see for example Fig. 9).

Figure 13(a) shows a 'scattergram' of the shear viscuit and the corresponding quantity for the potential energy, for two times, $t=0.078$ and 2.525. At short times there is a strong ('wishbone') correlation between the ST and the initial value of, $X(0)$. At longer times which corresponds to the transport coefficient relaxation time, the scattergram adopts a 'butterfly' shape (first reported for the viscuit in Ref. 25). The corresponding potential 
energy scattergram is statistically the same. Note that in both cases the distributions are very similar for the shear stress and potential energy derived quantities. This indicates that the potential energy landscape and its time evolution plays an important role in governing the transport coefficients. This is a well-known conclusion in the literature, which in fact is the basis of a number theories of the liquid state discussed in the Introduction. The kinetic contributions to the energy still have an indirect but important effect on the evolution of the potential energy with time even though the kinetic contribution to the transport coefficients is relatively small at liquid-like densities. Therefore it is necessary to consider the effects of the kinetic contributions to the energy to understand the origins of the transport coefficients.

Figure 13(b) shows the corresponding profiles for the supercooled liquid state D. The long time $(t=1.15$, here $)$ viscuit distributions have some similarities with those in frame (a). There are however a number of qualitative differences between those of the equilibrium state in frame (a). The ST distributions for the potential energy and stress are significantly more localized on the plane and to the left in frame (b), and the symmetry about ordinate axis has been lost. There is a significant bias towards negative viscuits, which indicates a frequent change in sign of the stress and excess energy. This trend is consistent with the picture that the configuration of particles is oscillating with small amplitudes about its potential minimum. Dissipative trajectories (i.e., the positive viscuits) still occur but are fewer and 'caged' intermittent events (this is consistent with the PDFs of Fig. 8). This suggests that the quench directs the system into one of the valleys on the landscape with depths lower than the kinetic energy. The evidence from the literature is that the arrangement of particles in such a basin is in a stressed state, ${ }^{52-55}$ and remains there for long enough to compute quasi-equilibrium data. ${ }^{56,57}$ At short time there is a stronger correlation between the initial value of stress and $u$ and the corresponding ST. 


\section{The potential energy landscape and the Fluctuation Theorem}

The present results for the ST PDFs bear striking similarites with those associated with the entropy production fluctuation theorems, ${ }^{58-60}$ (FT) whose general form is,

$$
\frac{P\left(\sigma_{t}\right)}{P_{t}\left(-\sigma_{t}\right)}=e^{\sigma_{t}}
$$

where $\sigma_{t}$ is the net entropy production over time $t$ due to an applied field. Equation (28) is valid for systems driven arbitrarily far from equilibrium. Equation (25) is of the same form as Eq. (28) which was confirmed numerically in Fig. 10. In the case of constant magnitude planar Couette flow, $\sigma_{t}=-\left[V / k_{B} T\right] \dot{\gamma} \int_{0}^{t} P_{y z}(x) d x$, whereas in the ST context the argument of the exponential is proportional to the viscuit. Another difference is that the system considered here is at equilibrium, when the right hand side of Eq. (28) is unity. There is no external field in the present work such as might be introduced by Non-equilibrium Molecular Dynamics (NEMD). ${ }^{61}$ The viscuit formulation is essentially a rewriting of the Green-Kubo formula where the entropy producing and decreasing trajectories which would be produced on the applicaton of a small field, have been separated into different PDFs. For the viscuit PDFs the asymmetry in the PDF between the negative and positive sides of the distribution is due to short term correlation between successive values of a variable. From an initial state, subsequent states are more likely to have a property value of the same sign and magnitude as the initial one, a correlation which decays with time. This will lead to more of the trajectories being 'entropy producing' than entropy absorbing on the application of a small external field to the system.

In contrast, in the limit of small applied fields the PDF involved in the FT tends to a shifted gaussian where the negative and positive sides are symmetric. Considering the shear stress example, this PDF can be expressed in terms of the viscuits as follows (see Refs. ${ }^{62-65}$ for the 
key steps and derivations). Let $\sigma_{t}$ be the average of the shear stress, $\sigma$, over a time period, $t$,

$$
\sigma_{t}=\frac{1}{t} \int_{0}^{t} \sigma(x) d x
$$

which may also be known as the block average. The two PDFs of relevance are,

$$
P\left( \pm \sigma_{t}\right)=\frac{1}{\sqrt{2 \pi} \sigma_{d, t}} \exp \left(-\frac{\left( \pm \sigma_{t}-\bar{\sigma}\right)^{2}}{2 \sigma_{d, t}^{2}}\right)
$$

where $\bar{\sigma}$ is the average of $\sigma$ over the entire time domain covered and $\sigma_{d, t}$ is the standard deviation of the block averages. See the supplementary material for the proof that this shifted gaussian satisfies the FT. For large $t$,

$$
\begin{aligned}
\left(\sigma_{t}-\bar{\sigma}\right)^{2} & \simeq \frac{2}{t} \int_{0}^{t}<\delta \sigma(0) \delta \sigma(t)>d s \\
& =\frac{2}{t}<\int_{0}^{t} \delta \sigma(0) \delta \sigma(t)>d s \\
& =\frac{2}{t}<\delta \sigma(0) \int_{0}^{t} \delta \sigma(t)>d s \\
\lim _{\dot{\gamma} \rightarrow 0}\left(\sigma_{t}-\bar{\sigma}\right)^{2} & =\frac{2}{t}<X_{u, t}>
\end{aligned}
$$

where $\delta \sigma(t)=\sigma_{t}-\bar{\sigma}$ and $X_{u, t}$ is the viscuit. Inspection of the formulas for the FT and viscuit PDFs in Eqs. (30) and (31), respectively, show that they are not obviously related, as the FT PDF involves an average of the viscuit and the viscuit is a function of the system in the zero shear rate limit. Nevertheless the strong similarity in the behavior of the two different types of PDF suggests that there may be a more direct formal link which connects the viscuit PDFs and those average quantities which comply with the FT in the zero field limit. ${ }^{62}$ The smaller the field the closer the FT PDF approaches a gaussian. In the GK-viscuit PDFs the (virtual) field can be made arbitrarily small without changing the relative probability of entropy increasing to entropy decreasing trajectories (i.e., is independent of field strength).

The viscuit PDF, in contrast, has a nongaussian form, based on exponentials, and this is in the zero shear rate limit. It is not obvious how to combine these two trends in a 'unified' theory. 
The less frequent larger viscuits may come from more structured parts of the potential energy landscape, while the cusp-like region near the origin of the PDF would come from the flatter regions of the PEL where there are more low activation energy avenues for stress relaxation. This is illustrated in the schematic diagram in Fig. 14. Although a completely different field, these ideas parallel those developed to account for interfacial separation of rough solid surfaces under load by Persson. ${ }^{66,67}$ The load pressure takes the place of the PDF and the separation, that of the viscuit. Assuming a fractal surface roughness, the theory produces an exponential tail in the low deformation limit, and as the pressure increases the asperities become more deformed and the surface smoother (this is the cusp region). Exponential (activation energy) terms appear in kinetic models of diffusion in liquids, ${ }^{68}$ and these may be considered to play an important role in governing the form of the viscuit PDFs. 


\section{CONCLUSIONS}

Equilibrium state point transport coefficients of liquids have traditionally been determined by, for example, the Green-Kubo (GK) technique. This involves integrating with time a time origin-averaged time correlation function. A complementary approach was introduced in Ref. 25, which reveals new aspects of the factors that determine a transport coefficient. This procedure is to simply reverse the order of the integral and time-origin averaging in GK which leads to the transport coefficient being the first moment of a probability distribution function of so-called single trajectory (ST) dynamical and structural events. We referred to these events as 'viscuits' in Ref. 25 in which the shear viscosity was investigated, and is used here as a general term for the corresponding properties of other quantities (transport and thermodynamic). This is information that is already available in the GK computational procedure, but is not usually analysed for its statistical properties.

The main advantage of the viscuit analysis, in our opinion, is it provides another 'window' to understand the factors that determine the origins of the viscosity and other transport coefficients. In particular it could provide insights into the role played by the time dependence of the potential energy landscape on the transport coefficients. This link is approximated in a number of prominent theories of the dynamics of liquid state, and the present approach offers the possibility of adopting a more systematic methodology for quantifying this link (and hence improving these theories). The viscuit values added up give the same value for the transport coefficient as the GK approach, as the two approaches are formally the same in that respect. There may be practical benefits in using the viscuit PDF approach compared to GK, for example, in 'filtering out' viscuit ranges that are noisy and contribute relatively little to the value of the transport coefficient, but this would need further exploration. 
This work also considers the bulk viscosity, thermal conductivity and self-diffusion coefficient. The sensitivity of the PDFs to state point in equilibrium and metastable liquids is also investigated. Positive values of these quantities are in all cases found to be associated with PDFs that are asymmetric, with the negative viscuit ('entropy absorbing') side being more 'peaky' and short-ranged near the origin than on the positive viscuit side. This follows from Eq. (17) which shows that the height of the PDF on each side is inversely proportional to its standard deviation. The standard deviation is larger on the positive side as positive viscuits are overall more probable (hence a larger standard deviation) because property values are correlated and similar for a few successive time steps. It was found that the two PDFs are statistically the same on reflection about the ordinate axis when the viscuit argument of the PDF is normalized by its standard deviation (determined separately for the two sides), provided the time in the viscuit definition is not excessively short compared to the correlation time of the associated time correlation function in the Green-Kubo treatment. The differences between the two sides of the PDF are then due solely to the differences in their respective standard deviations. Another surprising property of this 'reduced' form of probability distribution function ('PDFR') is that it is statistically independent of system size, transport coefficient and state point, providing the system is in the equilibrium fluid range. The underlying causes of this remarkable insensitivity to system specification are not known as far as we are aware. We also carried out simulations with a purely repulsive inverse power poential with an exponent of 12 at several state points in the equilibrium fluid part of its phase diagram. It was found that the PDFR of this system was also independent of state point and is the same as that of the LJ system. Further investigation would be required to see if this is also the case for other potentials, such as those that have, for example, an important coulombic component, and also for polyatomic molecule systems. 
The PDFR can be represented very well by a sum of several exponentials. Significant contributions to the value of the transport coefficient come from many standard deviations from the mean (e.g., up to almost about 8). The practically important large viscuits are extreme rare flow events which could imply slowly decaying states originating in highly structured parts of the potential energy landscape (see the schematic in Fig. 14). This is consistent with the observation by simulation that the high wavevector components of the shear stress autocorrelation function are more slowly decaying with time. ${ }^{69} \mathrm{~A}$ large viscuit could be due to a large instantaneous modulus or a long relaxation time, or a combination of the two.

The equivalents to the viscuits for thermodynamic properties (e.g., the potential energy) were also computed, and found to be statistically indistinguishable from those of the transport coefficients. It is already known that the potential energy landscape underpins the transport coefficients, although there are still uncertainties on the details of this. The present results demonstrate through the viscuit analysis that their is a commonality between all the transport coefficients and the potential energy as they have same probability distribution function in reduced form for the systems studied in this work. Although the kinetic contribution to this behavior contributes little directly to the transport coefficients at liquid-like densities its role in driving the time dependence of the potential energy landscape and therefore the transport coefficients should not be underestimated. The simplicity of this result gives encouragement that more realistic models for the transport coefficients could be derived within the theoretical framework established in this study.

The PDFRs of out-of-equilibrium supercooled high density states do not exhibit the symmetry and 'universality' found for the equilibrium states. The asymmetry in the negative and 
positive viscuit regimes revealed in the PDFs and viscuit scattergrams suggest that these measures could be useful in exploring the behavior of supercooled and highly pressurized liquids important for rheology and tribology. An analytic formula for the PDF based on a partially-correlated stochastic approximation is shown not to fit the MD-generated PDFs very well, highlighting the need to account for the classical equations of motion in any model of these event processes.

\section{SUPPLEMENTARY MATERIAL}

The numerical data for calculated properties of state points A-C is presented in tabular form in supplementary material. Another simple derivation of the FT for a near equilibrium sheared system represented by a gaussian PDF is presented (this result is already well-known in the literature, $\left.{ }^{62,63}\right)$.

\section{ACKNOWLEDGMENTS}

DD and DMH would like to acknowledge the support received from the EPSRC under the Established Career Fellowship Grant No. EP/N025954/1. All data can be made available by emailing the authors of the paper or tribology@imperial.ac.uk. 


\begin{tabular}{ccccccc}
\hline Key & $T$ & $\rho$ & $\eta_{s}$ & $\eta_{b}$ & $\lambda$ & $D$ \\
\hline A & 2.0 & 0.5000 & $0.627(1)$ & $0.39(1)$ & $2.80(1)$ & $0.399(2)$ \\
B & 1.0 & 0.8000 & $2.09(1)$ & $0.87(1)$ & $6.68(1)$ & 0.067 \\
C & 0.722 & 0.8442 & $3.28(1)$ & $1.22(1)$ & $6.90(1)$ & $0.032(1)$ \\
D & 0.500 & 0.9211 & 1688 & $2.62(5)$ & $9.29(2)$ & $0.0(1)$ \\
\hline
\end{tabular}

TABLE I: The transport coefficients for the four state points considered in this study. States A-C are in the equilibrium fluid part of the Lennard-Jones phase diagram. State D is an out-of-equilibrium metastable state in the supercooled part of the LJ phase diagram, ${ }^{36}$ which explains the extreme values of the TC compared with those of states A-C. 


\begin{tabular}{ccccc}
\hline Quantity & $\eta_{s}$ & $\eta_{b}$ & $\lambda$ & $D$ \\
\hline$X_{D,-}$ & $-4.432(9)$ & $-2.00(2)$ & $-11.64(3)$ & $-0.0942(1)$ \\
$X_{D,+}$ & $7.72(3)$ & $3.18(5)$ & $18.55(4)$ & $0.1261(1)$ \\
$X_{D}$ & $3.29(2)$ & $1.18(4)$ & $6.91(3)$ & $0.03194(9)$ \\
$X_{G K}$ & $3.29(2)$ & $1.20(4)$ & $6.94(3)$ & $0.03193(5)$ \\
$X_{P,-}$ & $-4.432(9)$ & $-2.00(2)$ & $-11.64(3)$ & $-0.0942(1)$ \\
$X_{P,+}$ & $7.72(3)$ & $3.18(5)$ & $18.55(4)$ & $0.1262(5)$ \\
$X_{P}$ & $3.29(2)$ & $1.18(4)$ & $6.91(3)$ & $0.0320(2)$ \\
$\sigma_{-}$ & $12.04(3)$ & $5.29(6)$ & $31.06(9)$ & $0.2495(9)$ \\
$\sigma_{+}$ & $16.96(5)$ & $7.1(1)$ & $41.3(1)$ & $0.299(1)$ \\
$\sigma_{+} / \sigma_{-}$ & $1.4086(4)$ & $1.342(7)$ & $1.330(4)$ & $1.198(2)$ \\
$N_{+} / N_{-}$ & $1.252(2)$ & $1.208(5)$ & $1.207(1)$ & $1.1252(7)$ \\
$I_{N}$ & $0.807(2)$ & $0.807(6)$ & $0.814(3)$ & $0.781(4)$ \\
$I_{\sigma}$ & $0.670(3)$ & $0.664(9)$ & $0.677(5)$ & $0.646(5)$ \\
\hline
\end{tabular}

TABLE II: Computed quantities for state point C using NVE dynamics, in LJ units or dimensionless. The duration $t=2.49$ is the integration time for GK and the ST. The properties, $X_{D,-}$ and $X_{D,+}$ are the values of the transport coefficient derived by summing the negative and positive ST values, respectively, and $X_{D}$ is the total value. $X_{G K}$ is the value of the transport coefficient obtained from the Green-Kubo formula. $X_{P,-}, X_{P,+}$ and $X_{P}$ are the components and total value of the transport coefficient obtained by numerical integration of the ST PDF. These quantities are defined in Eqs. (3), (6), (9), and (10), for $\eta_{s}, \eta_{b}, \lambda$ and $D$ respectively. The quantities, $\sigma_{-}$and $\sigma_{+}$are the ST standard deviations of the negative and positive sides of the PDF. The ratio, $N_{+} / N_{-}$, is the number of entrants on the positive side divided by those on the negative side. The quantity, $I_{N}$ is defined in the last line of Eq. (14),

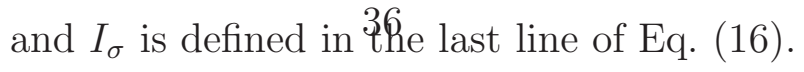




\section{References}

$\dagger$ Electronic address: d.heyes@imperial.ac.uk

$\ddagger$ Electronic address: d.dini@imperial.ac.uk

$\S$ Electronic address: Edward.Smith@brunel.ac.uk

1 M. Goldstein, J. Chem. Phys. 51, 3728 (1969).

${ }^{2}$ H. Spikes and Z. Jie, Tribol. Lett. 561 (2014).

${ }^{3}$ R. Zwanzig, J. Chem. Phys. 79, 4507 (1983).

4 T. Keyes, J. Chem. Phys. 103, 9810 (1995).

5 J.C. Dyre and N.B. Olsen, Phys. Rev. A 69, 042501 (2004).

6 J.C. Dyre and W.H. Wang, J. Chem. Phys. 136, 224108 (2012).

7 U. Buchenau, J. Chem. Phys. 134, 224501 (2011).

8 F.H. Stillinger and T.A. Weber, Phys. Rev. A 28, 2408 (1983).

9 B. Madan, T. Keyes and G. Seeley, J. Chem. Phys. 92, 7565 (1997).

10 S. Chowdhury, S. Abraham, T. Hudson and P. Harrowell, J. Chem. Phys. 144, 124508 (2016).

11 J.D. Gezelter, E. Rabani and B.J. Berne, J. Chem. Phys. 107, 4618 (1997).

12 T. Keyes, G.V. Vijayadamodar and U. Zurcher, J. Chem. Phys. 106, 4651 (1997).

13 W.-X. Li, T. Keyes, R.L. Murry and J.T. Fourkas, J. Chem. Phys. 109, 9096 (1998).

14 E.D. Chisolm and D.C. Wallace, J. Phys.: Cond. Matt. 13, R739 (2001).

15 E.D. Chisolm, B.E. Clements and D.C. Wallace, Phys. Rev. E 63, 031204 (2001).

16 P. Sollich, F. Lequeux, P. Hébraud and M.E. Cates, Phys. Rev. E 78, 2020 (1997).

17 P. Sollich, Phys. Rev. E 58, 738 (1998). 
18 S.M. Fielding, P. Sollich and M.E. Cates, J. Rheol. 44, 323 (2000).

19 J.J. Erpenbeck, Phys. Rev. E 51, 4296 (1995).

20 S. Hess and D.J. Evans Phys. Rev. E 64, 2514 (2001).

21 S. Viscardy and P. Gaspard, Phys. Rev. E 68, 041204 (2003).

22 S. Viscardy, J. Servantie, and P. Gaspard, J. Chem. Phys. 126, 184513 (2007).

23 F. H. Stillinger and P. G. Debenedetti, J. Phys. Chem. B 109, 6604 (2005).

24 M.S. Shell, F. H. Stillinger and P. G. Debenedetti, J. Phys. Condens. Matt. 17, S4035 (2005).

25 D.M. Heyes, E.R. Smith and D. Dini, J. Chem. Phys. 150, 174504 (2019).

26 J.-P. Hansen and I.R. McDonald, Theory of simple liquids, (Academic Press, Amsterdam, 2006) 4th. ed.

27 E. Helfand, Phys. Rev. 119, 1 (1960).

28 J.A. McLennan, Prog. Theor. Phys. 30, 408 (1963).

29 D. M. Heyes J. Chem. Soc. Farad. Trans. II, 80, 1363 (1984).

30 P.L. Palla, C. Pierleoni, G. Ciccotti, Phys. Rev. E 78, 021204 (2008).

31 H. Okumura and F. Yonezawa, J. Chem. Phys. 116, 7400-7410 (2002).

32 H. Okumura and F. Yonezawa, Phys. Rev. E 67, 021205 (2003).

33 D.M. Heyes and J.G. Powles, Mol. Phys. 99, 1077 (2001).

34 A.E. Nasrabad, N.M. Oghaz, and B. Haghighi, J. Chem. Phys. 129, 024507 (2008).

35 D.M. Heyes, The liquid state - applications of molecular simulations (Wiley, Chichester, 1998).

36 J.H.R. Clarke, J.Chem. Soc. Farad. Trans. II 75, 1371 (1979).

37 D. Levesque and L. Verlet, Phys. Rev. A 2, 2514 (1970).

38 D. Levesque, L. Verlet and J. Kürkijarvi, Phys. Rev. A 7, 1690 (1973).

39 W.G. Hoover, Phys. Rev. A 31, 1695 (1985).

40 M.P. Allen and D.J. Tildesley, Computer simulation of liquids (Oxford UP, Oxford, 2017). 
41 A. Greco, W.H. Matthaeus, S. Servido and P. Dmitruk, Phys. Rev. E 80, 046401 (2009).

42 J.C. Phillips, Rep. Progr. Phys. 591133 (1996).

43 C.P. Lindsey and G.D. Patterson, J. Chem. Phys. 73, 3348 (1980).

44 R. Zorn, J. Chem. Phys. 116, 3204 (2002).

45 E. Rabani, J.D. Gezelter and B.J. Berne, Phys. Rev. Lett. 82, 3649 (1999).

46 M.O. Vlad, D.L. Huber and J. Ross, J. Chem. Phys. 106, 415 (1997).

47 S.I. Simdyankin and N. Mousseau, Phys. Rev. E 68, 041110 (2003).

48 S. Nararajah and T.K. Pogány, C.R. Acad. Sci. Paris, Ser. 1, 354201 (2016).

49 G. Cui, X. Yu, S. Iommelli and L. Kong, IEEE Sign. Process. Lett. 231662 (2016).

50 J. Petravic, J. Chem. Phys. 121, 11202 (2004).

51 K. Broderix, K.K. Bhattacharya, A. Cavagna, A. Zippelius and I. Giardina, Phys. Rev. Lett. 85, $5360(2000)$.

52 S. Abraham and P. Harrowell, J. Chem. Phys. 137, 014506 (2012).

53 M.D. Ediger and P. Harrowell, J. Chem. Phys. 137, 080901 (2012).

54 I. Fuereder and P. Llg, J. Chem. Phys. 142, 144505 (2015).

55 S.R. Williams and D.J. Evans, J. Chem. Phys. 132, 184105 (2010).

56 T.B. Schrøder, S. Sastry, J.C. Dyre and S.C. Glotzer, J. Chem. Phys. 112, 9834 (2000).

57 B. Doliwa and A. Heuer, Phys. Rev. E 67, 030501 (2003).

58 D.J. Evans, E.G.D. Cohen, and G.P. Morriss, Phys. Rev. Lett. 71, 2401 (1993).

59 D. Evans and D. Searles, Phys. Rev. E 50, 1645 (1994).

60 G. Gallavotti and E. G. D. Cohen, Phys. Rev. Lett. 74, 2694 (1995).

61 D.J. Evans and G. Morris, Statistical Mechanics of Nonequilibrium Liquids, (Cambridge UP, Cambridge, 2008) 2nd. edit.

62 D.J. Searles and D.J. Evans, J. Chem. Phys. 112, 9727 (2000). 
63 D.J. Evans and D.J. Searles, Adv. Phys. 51, 1529 (2002).

64 A. Giuliani, F. Zamponi and G. Gallavotti, J. Stat. Phys. 119, 909 (2005).

65 E.M. Sevick, R. Prabhakar, S.R. Williams and D.J. Searles, Ann. Rev. Phys. Chem. 59, 603 (2008).

66 B.N.J. Persson, Phys. Rev. Lett. 99, 125502 (2007).

67 W.B. Dapp, N. Prodanov and M.H. Müser, J. Phys.: Cond. Matt. 26, 355002 (2014).

68 E. Rabani, J.D. Gezelter and B.J. Berne, J. Chem. Phys. 107, 6867 (1997).

69 P.B. Visscher and W.T. Logan, Phys. Rev. B 42, 4779 (1990). 


\section{Figure Captions}

Figure 1: Frame (a) shows the time correlation function relating to the four transport coefficients in GK, using $\rho=0.8442$ and $T=0.722$. Frame (b) shows the corresponding time dependent transport coefficients, $X(t)$, defined in Eqs. (3), (6), (9), and (10), for $\eta_{s}, \eta_{b}, \lambda$ and $D$ respectively. The shear viscosity, thermal conductivity and self-diffusion coefficient functions were computed in constant $T$ simulations while those for the bulk viscosity were using NVE dynamics.

Figure 2: The single trajectory viscosity PDF in LJ units (frame (a)) defined in Eq. (17) and in units of the averaged viscosity (in frame (b)) defined in Eq. (18) for the state point, ('C' in Table I) $\rho=0.8442$ and $T=0.722$ with $t=2.5$. The differences between the PDFs on the negative $\left(P_{-}\right)$and positive $\left(P_{+}\right)$sides are shown in red. On the left or negative side, $P_{-}-P_{+}$ is shown, and on the right $P_{+}-P_{-}$is given.

Figure 3: The four transport coefficients in LJ units derived from the accumulated first moment of the PDF, $P$. The data is from a simulation for state point is $\mathrm{C}$ in Table I, taking $t=2.5$. The transport coefficients obtained using the GK formulas are indicated by horizontal lines. Scaling factors to aid clarity of the different curves are given on the figure.

Figure 4: The time dependent shear viscosity, $\eta_{s}(t)$, (frame (a)) and self-diffusion coefficient, $D(t)$, (frame (b)) for state point C. The figures also show the separate contributions from the ST on the negative and positive sides $\left(i . e ., \eta_{s,-}\right.$ and $\eta_{s,+}$ for the shear viscosity and $D_{-}$and $D_{+}$for the self-diffusion coefficient. The GK values for the total transport coefficient are also shown as a function of $t$ in each case. Note the log-lin scale. 
Figure 5: The shear viscosity standard deviation reduced PDF defined in Eq. (11) for $t=2.5$ and several system sizes using $\rho=0.8442$ and $T=0.722$. Frames (a) and (b) give $P_{S D}\left(\left|\eta_{s, u}\right| / \sigma_{d}\right)$ on lin-lin and lin-log scales respectively The numbers of particles in the MD cells are are given in the figure key.

Figure 6: The shear viscosity, $P$-type $P D F$ s on the negative and positive sides of the distribution are shown for different $t$ values, given on the figure. The sets of curves are shifted vertically to improve clarity.

Figure 7: The reduced PDF, $P$, for the shear viscosity compared with an empirical fit to the simulation data for $t=2.49$ using Eq. (20). The constants are, $A_{1}=0.92726, A_{2}=1.45707$ and $A_{3}=0.50139$, and $b_{1}=2.57893, b_{2}=27.86215$ and $b_{3}=0.87189$. Frames (a) and (b) are on lin-lin and lin-log scales, respectively. The full fit and just the slowest decaying term ('fit limiting') are shown on both frames. The PDF of the total energy per particle is also shown. The simulations were carried out at constant temperature.

Figure 8: The state point dependence of the viscuit PDF. Key: (State A) $\rho=0.5, T=2.0$ for $t=1.7782$, and (State B) $\rho=0.8, T=1.0$ for $t=2.1501$. (State C) $\rho=0.8442, T=0.722$ for $t=2.4855$, Frame (a) shows the data on a lin-lin scale, and frame (b) presents the same data on a lin-log scale, taking the argument as $\left|\eta_{s, u}\right| / \sigma_{d}$. The out-of-equilibrium State Point D (magenta and green symbols for the negative and positive PDF sides, respectively) shows a significant breakdown of the symmetry. The positive ST side of the PDF is highly oscillatory unlike that of the negative side.

Figure 9: The dimensionless argument PDF, $P$ for the four transport coefficients at the state 
point, $\rho=0.8442$ and $T=0.722$. Frames (a) and (b) have the ordinates on lin and log scales, respectively. All calculations were performed with NVE dynamics. On the figure, 'S' stands for the shear viscosity, ' $\mathrm{B}$ ' for bulk viscosity, ' $\mathrm{T}$ ' for the thermal conductivity and ' $\mathrm{D}$ ' for the self-diffusion coefficient. Data for $t=0.5$ and 2.5 are given on the figure. Frame (b) also has the mean-field prediction using Eq. (21), with three values of the correlation coefficient, $c$, which are specified on the figure.

Figure 10: The ratios, $R$, of the $P_{L J}$ PDFs for the same absolute values of the ST, and for different integraton times, $t$, indicated on the figure. Frame (a) is for the shear viscosity and frame (b) shows the $R$ data associated with the thermal conductivity ST.

Figure 11: Frame (a): The parameter $A(t)$ obtained directly from the MD data slopes of Fig. 10(a) and from Eq. (26), $A=\left(\sigma_{+}-\sigma_{-}\right) / \sigma_{+} \sigma_{-}$. Frame (b): The ratio $\sigma_{-} / \sigma_{+}$as a function of $N_{-} / N_{+}$for the four transport coefficients is shown. The linear regression fit to the abscissa values greater than 0.6 has an intercept of -0.361 and a slope of 1.3425 . The viscuit equivalents for the potential energy landscape $u$ and pressure, $P$ are also shown on frame (b).

Figure 12: Frame (a): The shear ('S') and potential energy ('u') time traces, with points separated by 4.2 reduced time units (also the viscuit time, $t$ ). State point C and NVT dynamics were used. The time trace of the local time-averaged quantity ('t-av') and ST or viscuit ('visc') are shown. Frame (b) is the PDF of the quantity. $X_{t}$, for property $X$ summed over time $t$ or $N_{t}$ time steps. The PDFs on the negative and positive ST sides treated separately are given on the figure. The shear stress ('S') and potential energy, $u$ are the quantities considered again. A comparison is made with a gaussian distribution. The MD PDF was rescaled onto an equivalent gaussian using, $P_{g}(x)=\sqrt{2 / \pi} \exp \left(-\left[x / \sigma_{g}\right]^{2} / 2\right) / \sigma_{g}$. The reduced 
PDF, $P_{g, M D}=g P_{M D}\left(\left[x / \sigma_{M D}\right] / g\right)$, where $g=\sqrt{\pi /(\pi-2)}$ is plotted on the figure.

Figure 13: Frame (a) shows a 'scatter' plot of $P_{x y}(0)$ and the average deviatoric potential energy per particle, $u(0)-\bar{u}$ against the corresponding ST quantity. Both quantities are normalized separately by their corresponding standard deviations. The standard deviation of $P_{x y}(0)$ is denoted by $\sigma_{x y}$. The state point is $T=0.722$ and $\rho=0.8442$. Data for two times, $t=0.078$ denoted by 'A', and $t=2.525$ denoted by 'B', are shifted vertically by -5 and +5 , respectively, for clarity. Key: shear stress (black star), potential energy (red open square) for $t=0.078$, and shear stress (blue filled in square), potential energy (green open circle) for $t=2.525$, Frame (b) as for frame (a) except that data from a supercooled liquid state simulation at $T=0.5$ and $\rho=0.9211$ are presented. The symbols, 'A' and 'B' correspond in this frame to $t=0.011$ and 1.15 , respectively.

Figure 14: Schematic diagram illustrating the effects of qualitatively different regions on the potential energy landscape on the viscuit PDFs (indicated by the arrows). The $6 N$ phase space vector is represented qualitatively on a $2 D$ diagram. 
(a)

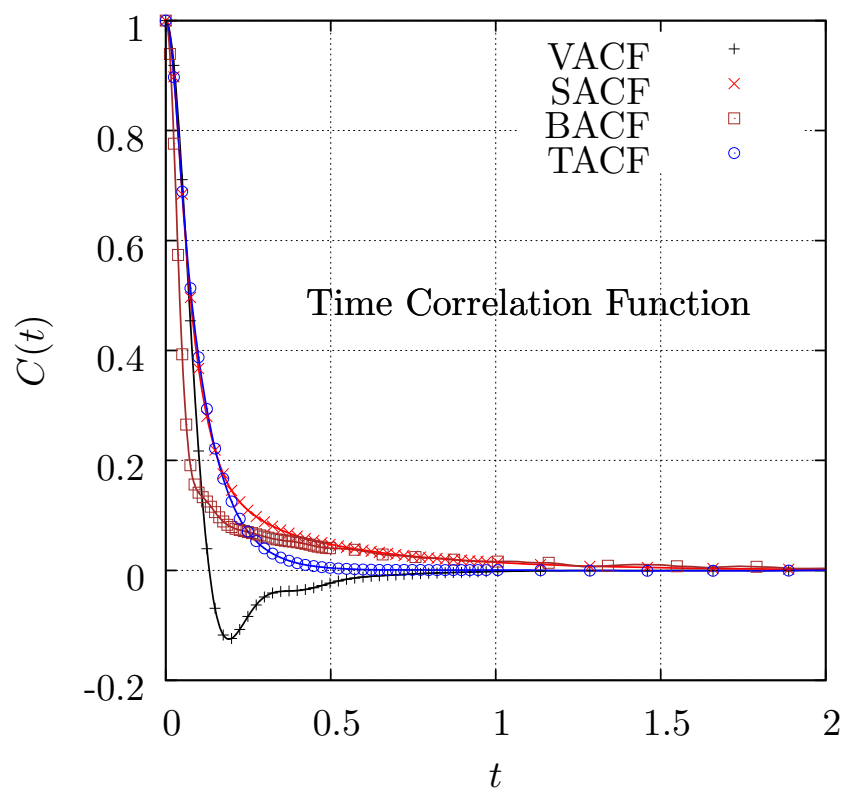

(b)

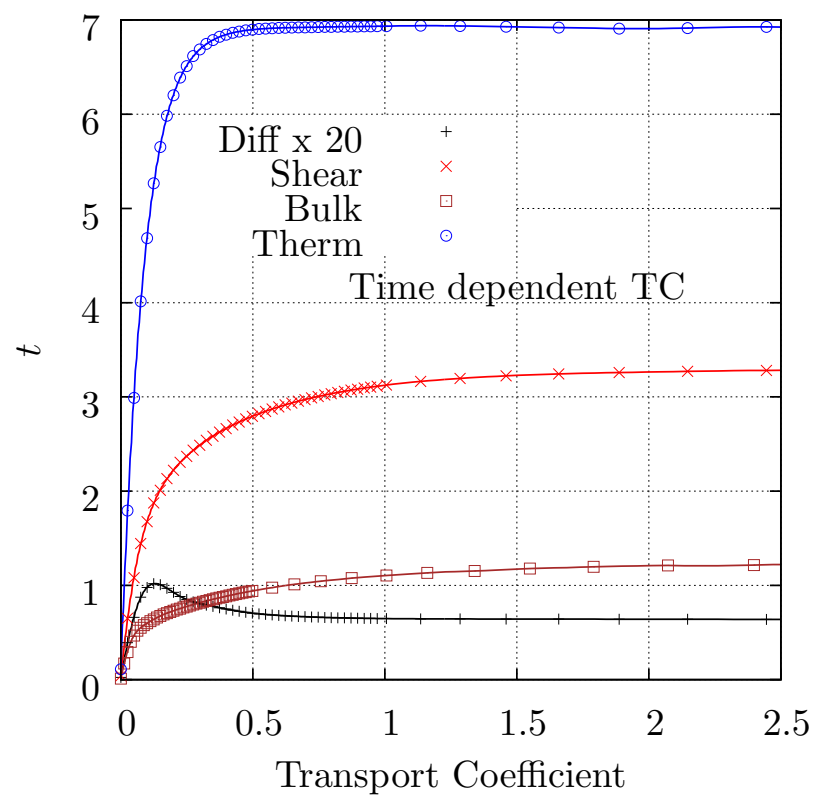

Figure 1 
(a)

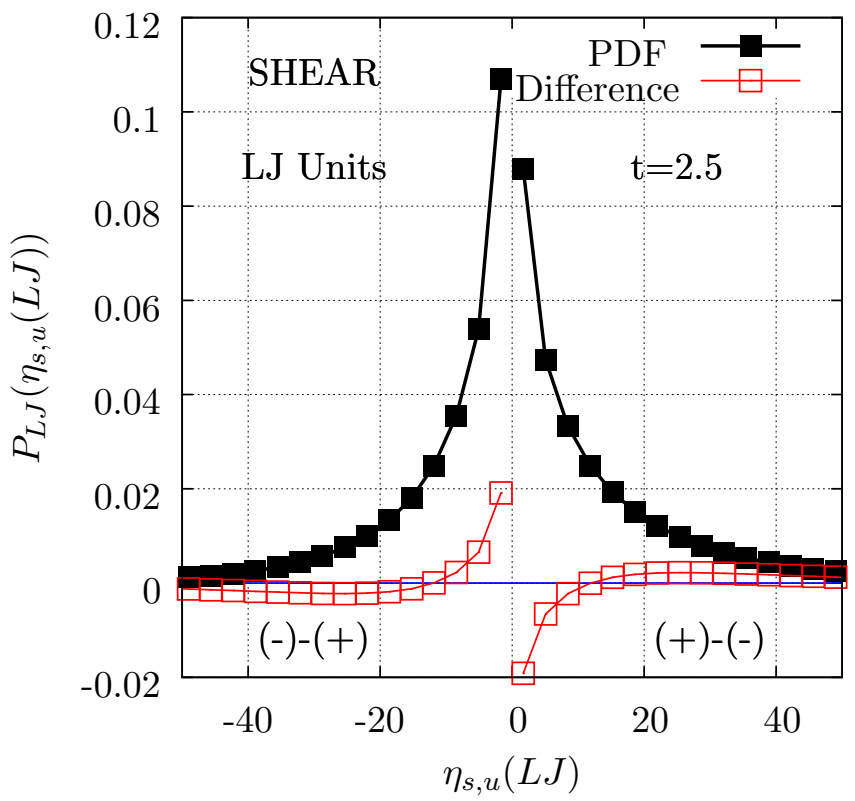

(b)

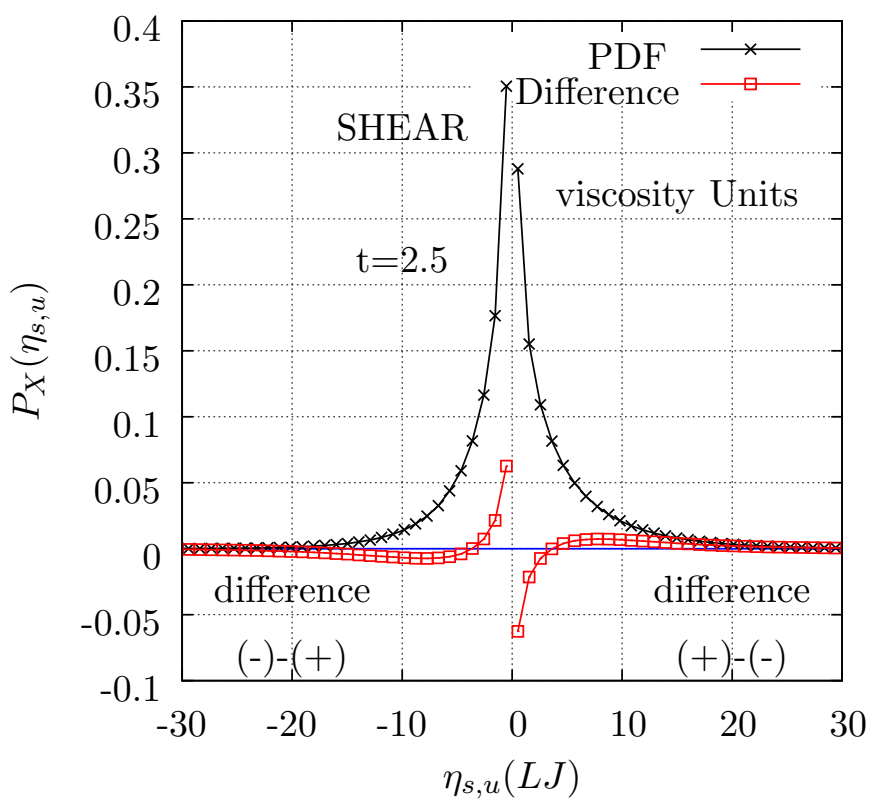

Figure 2 


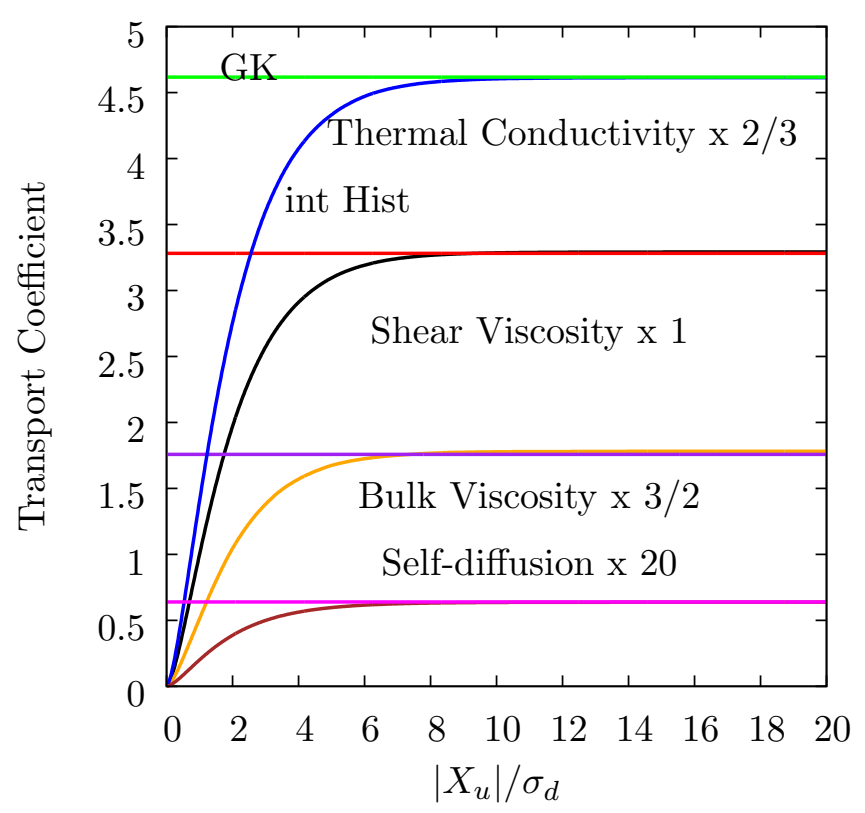

Figure 3 
(a)

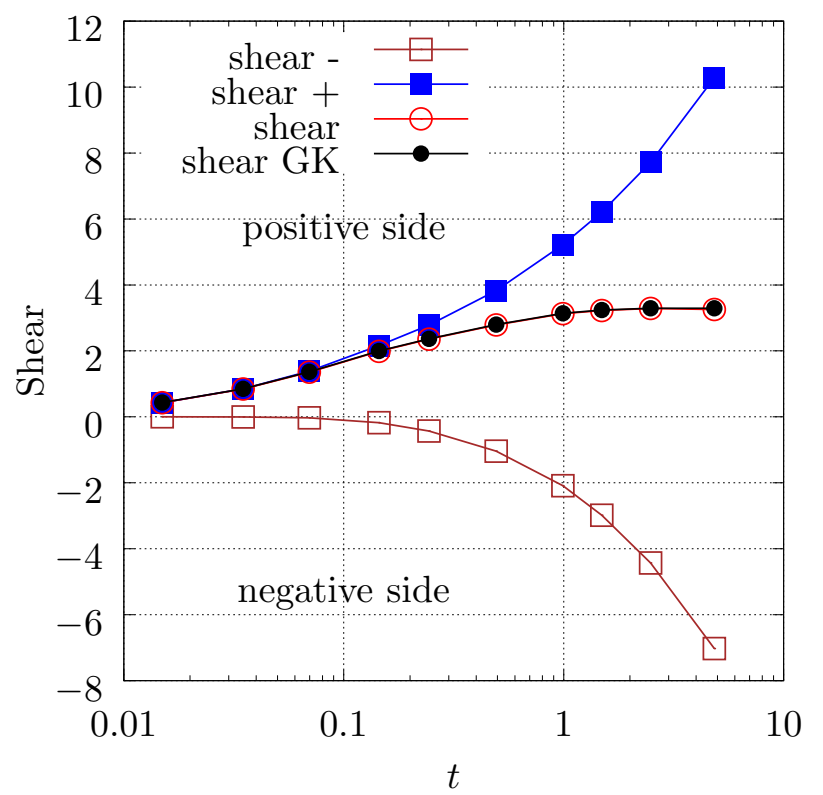

(b)

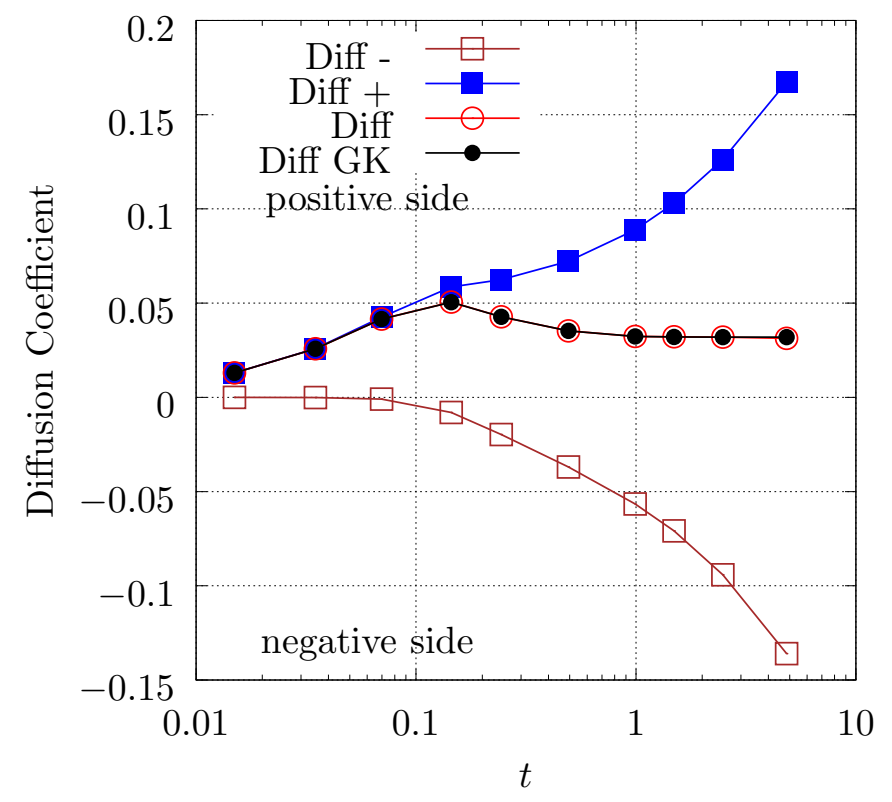

Figure 4 
(a)

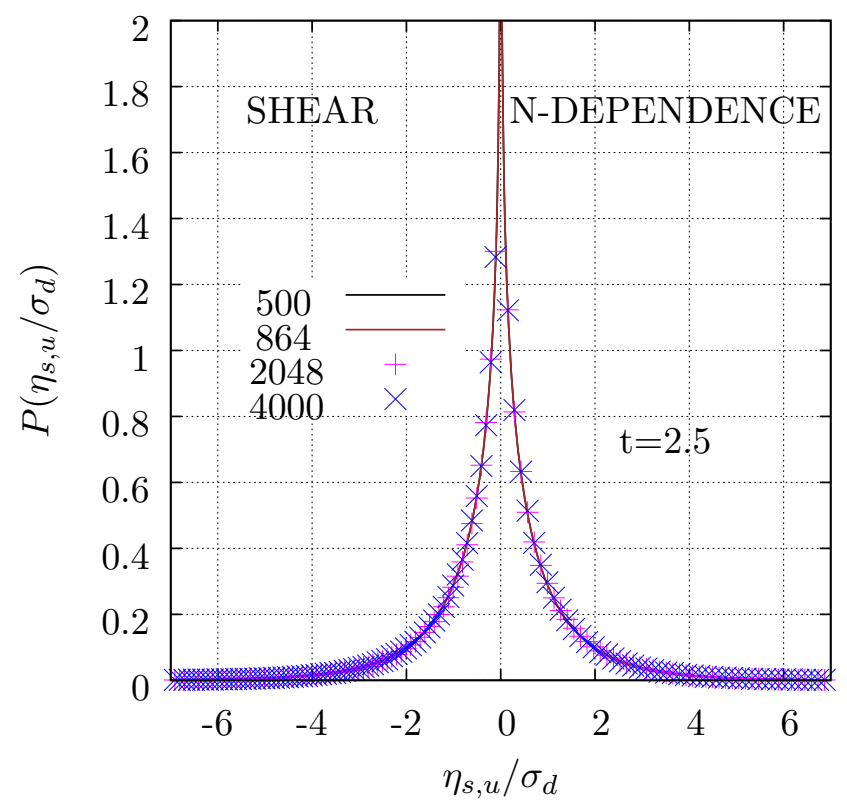

(b)

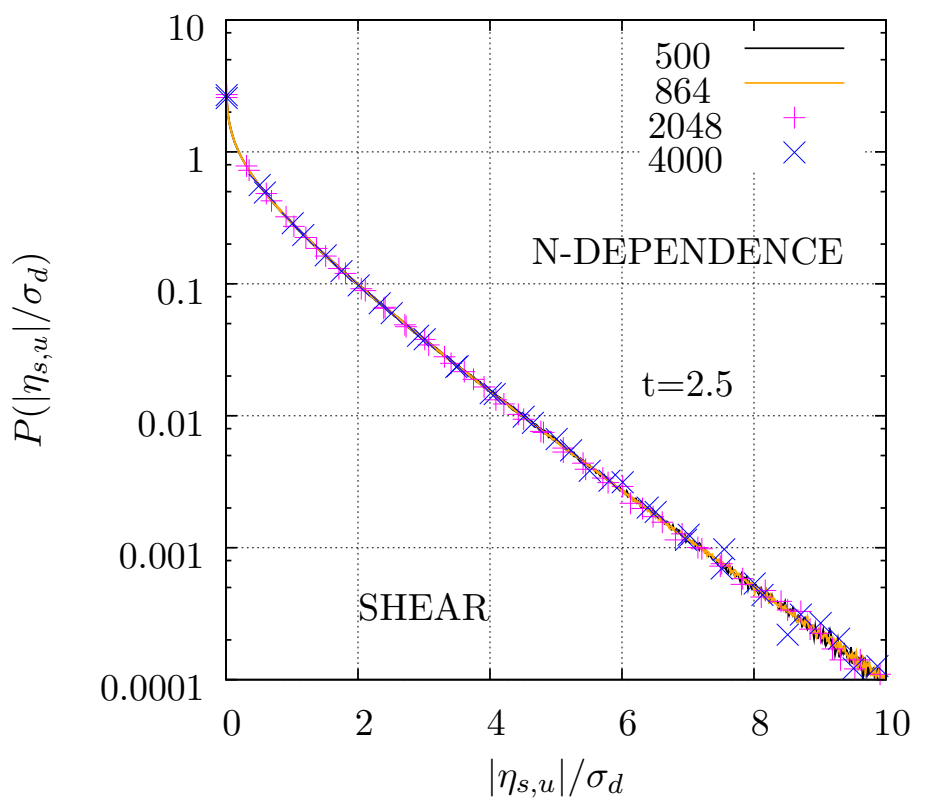

Figure 5 


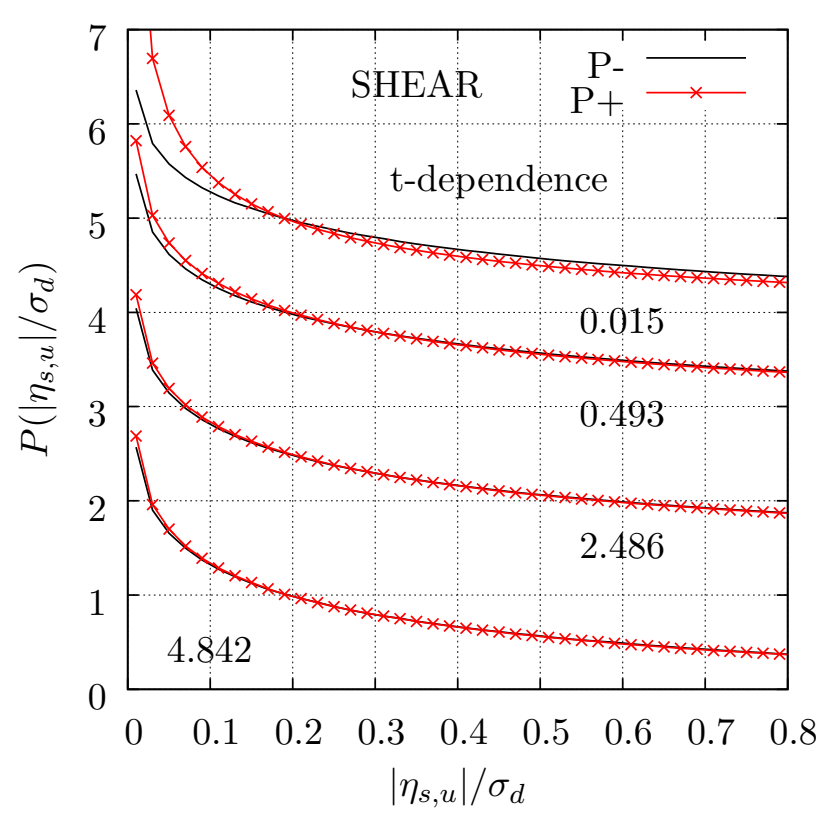

Figure 6 
(a)

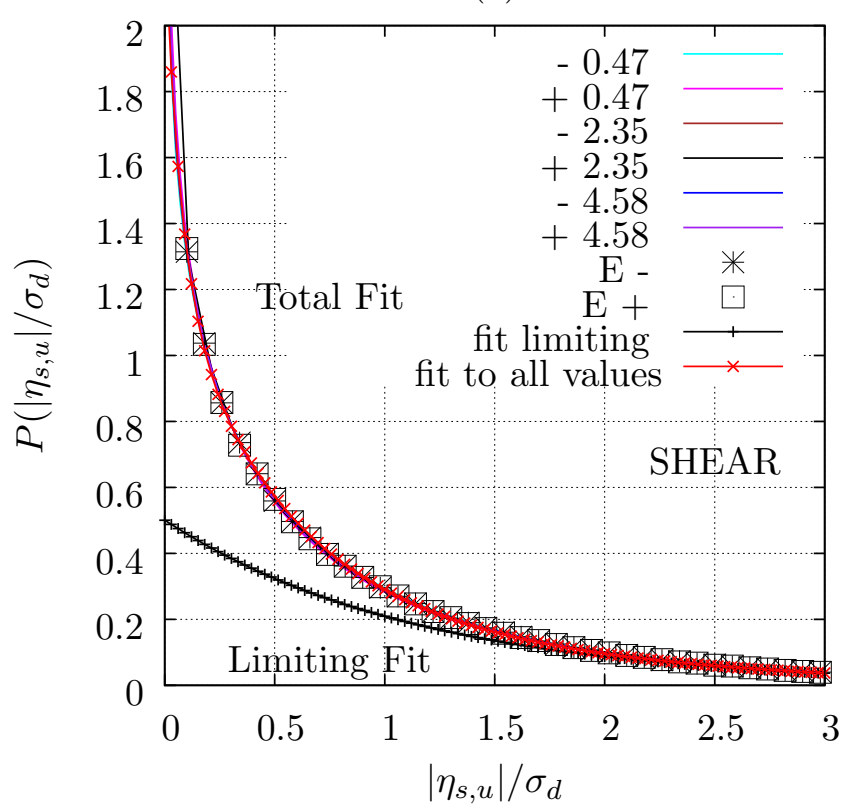

(b)

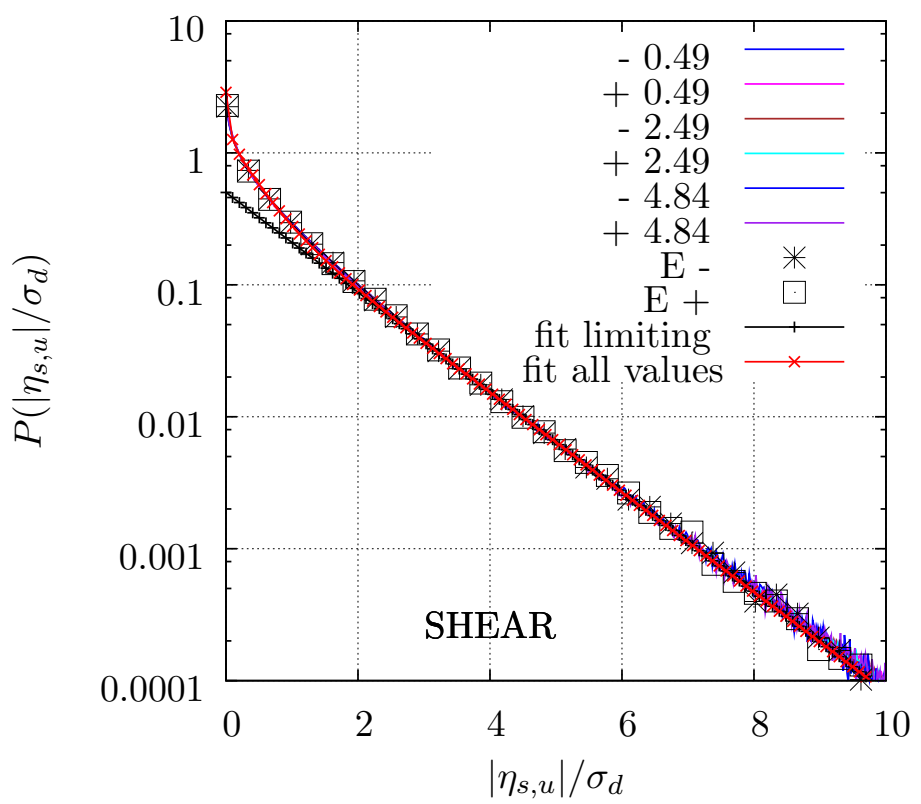

Figure 7 
(a)

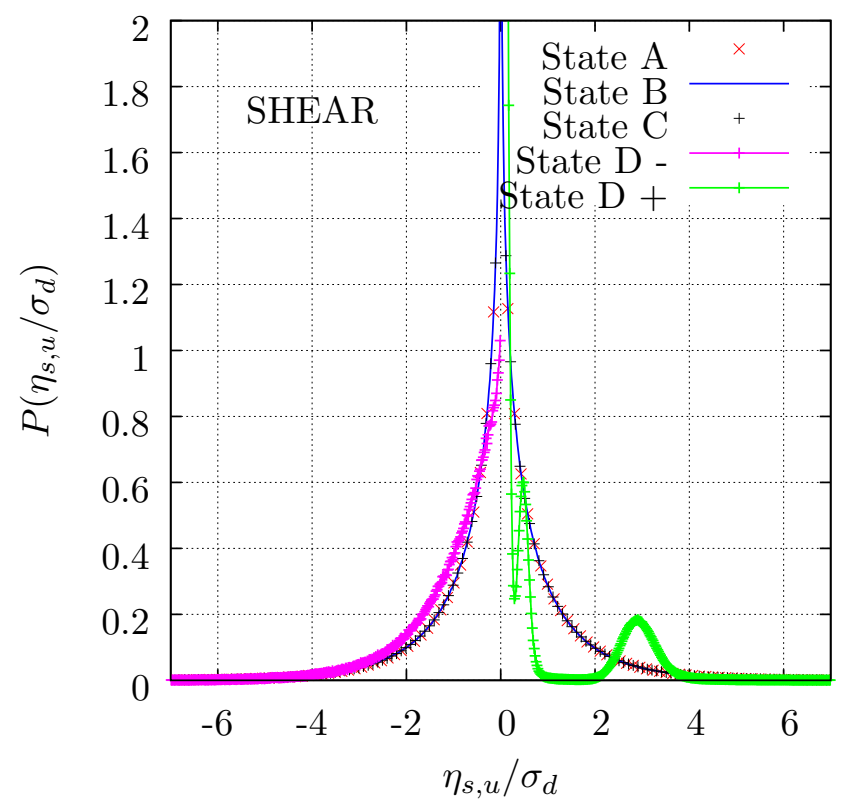

(b)

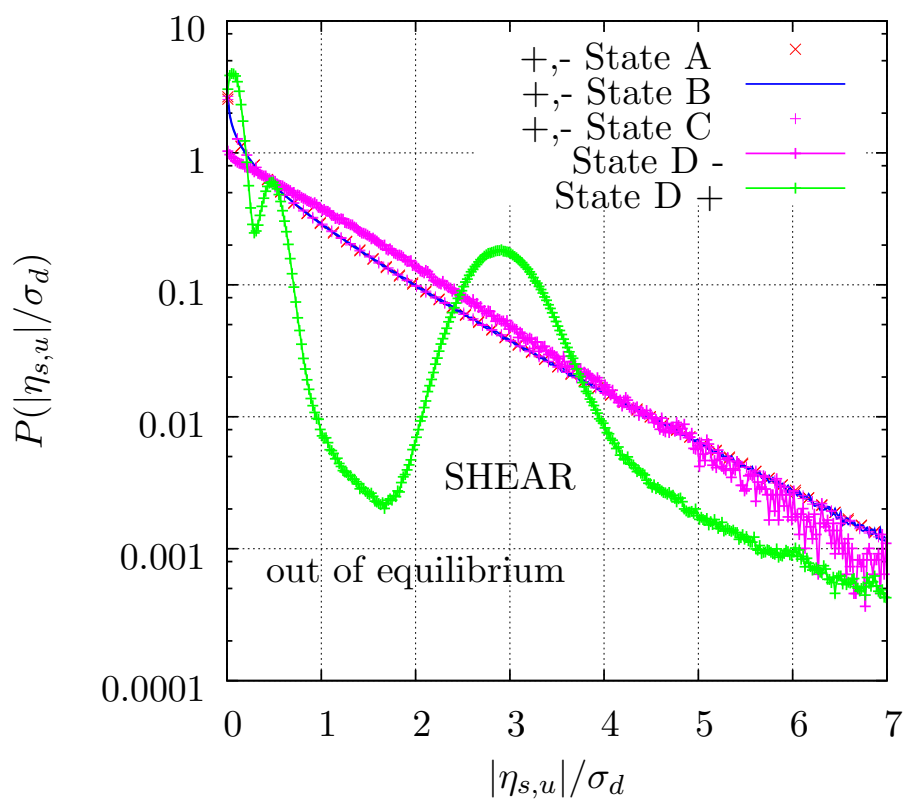

Figure 8 
(a)

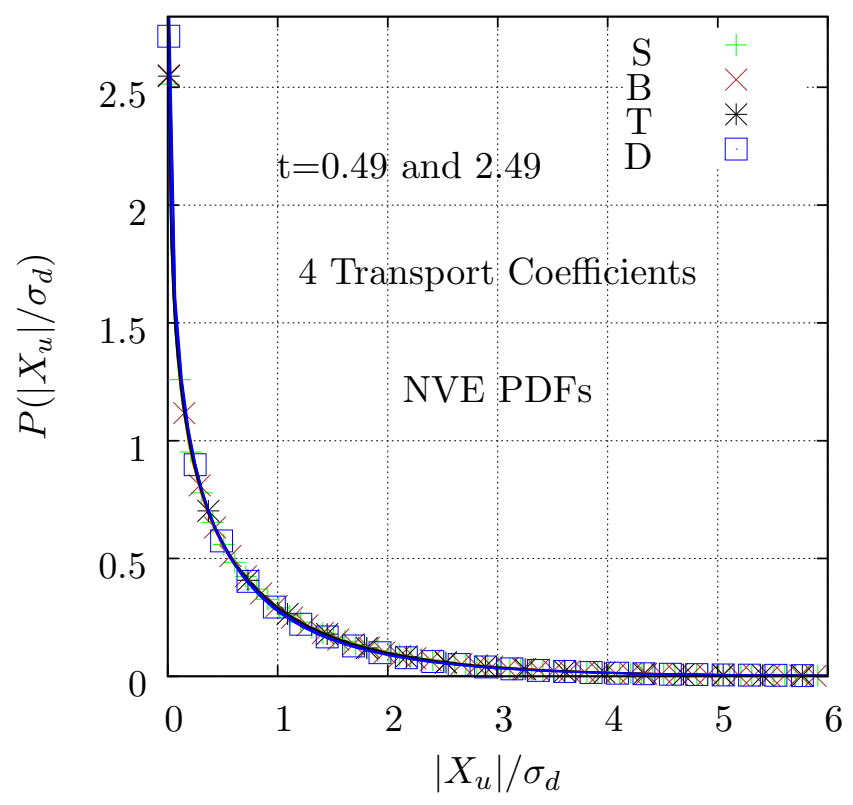

(b)

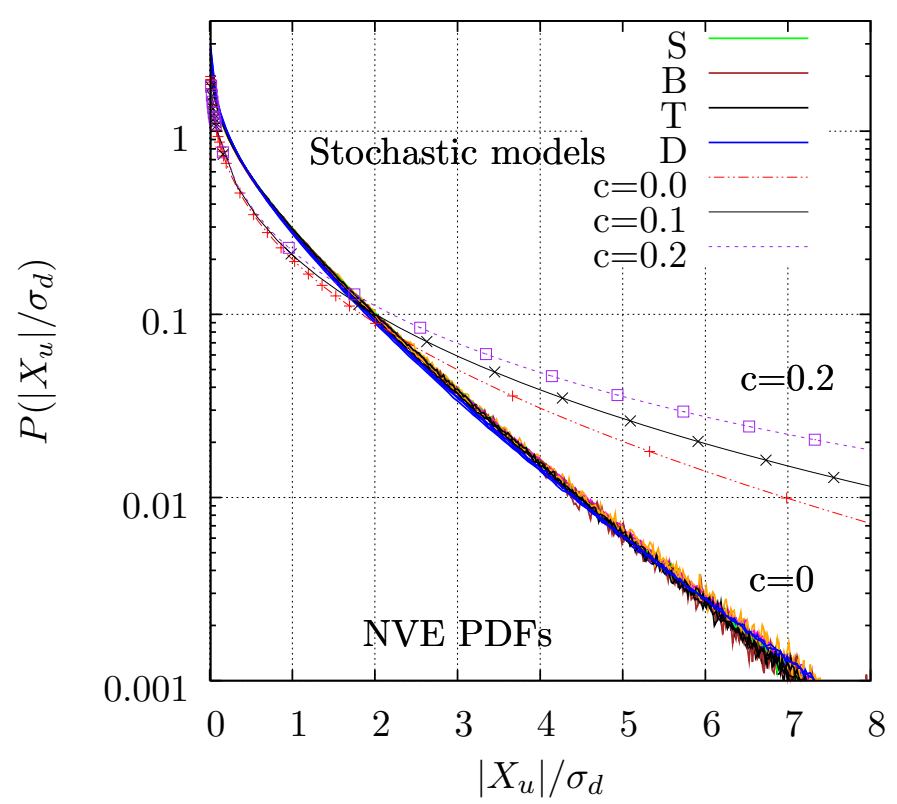

Figure 9 
(a)

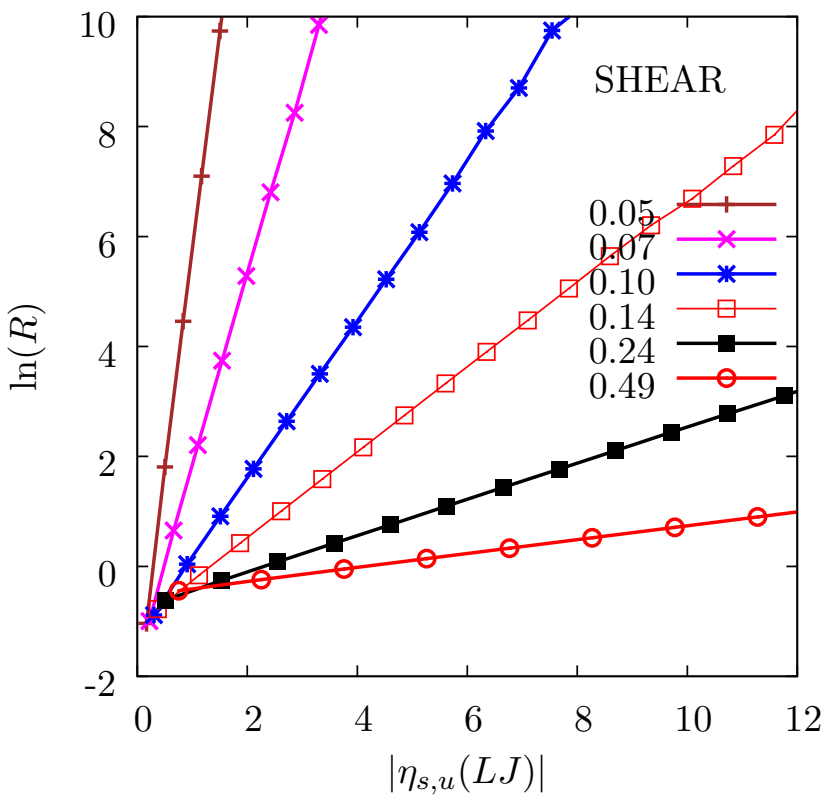

(b)

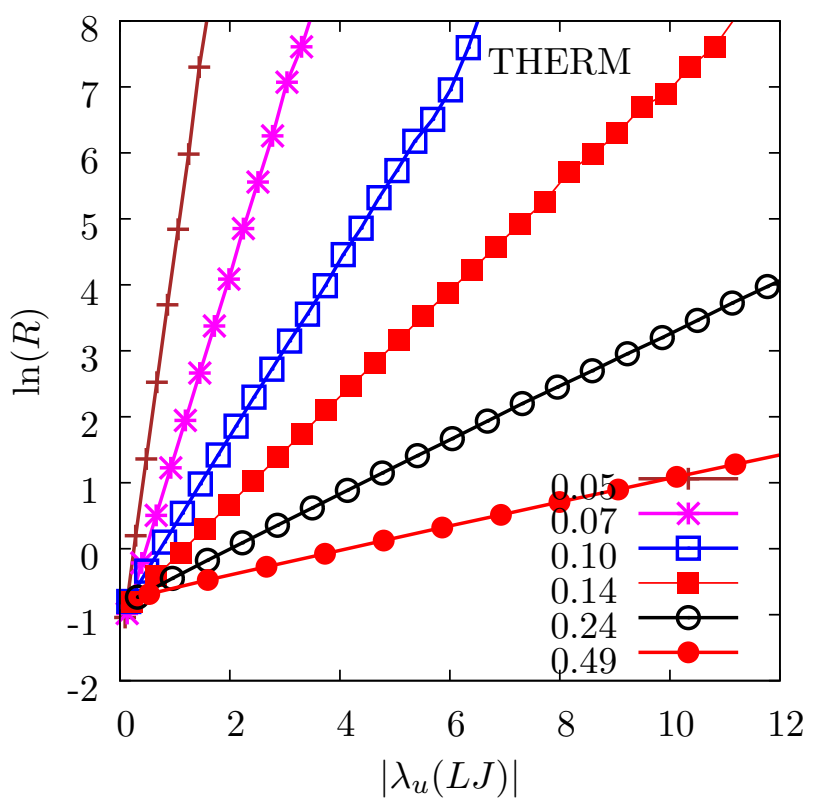

Figure 10 
(a)

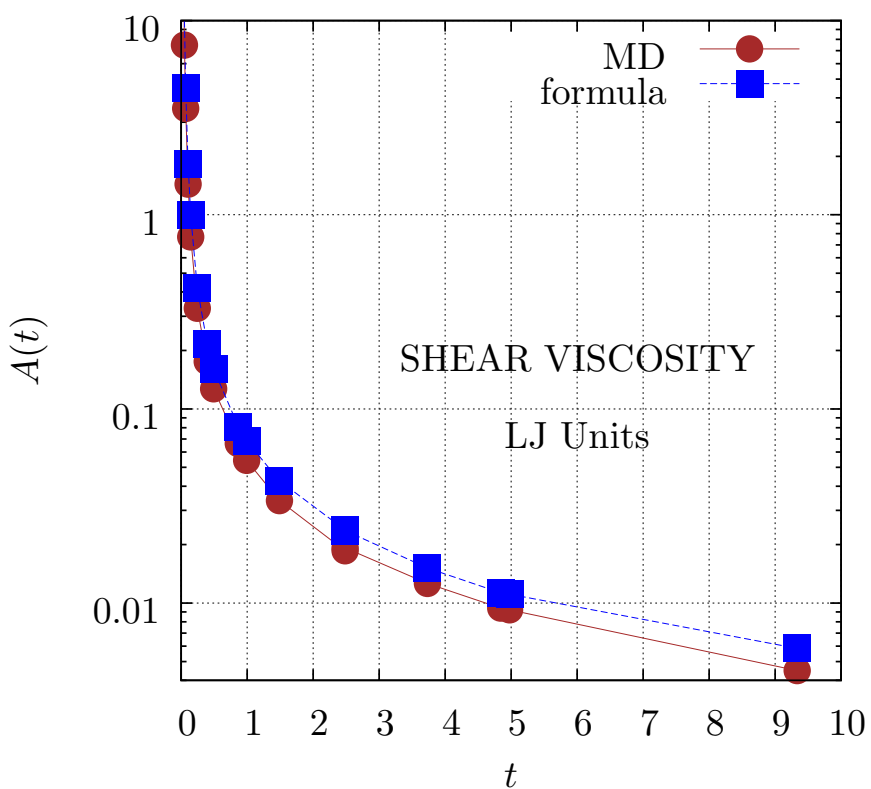

(b)

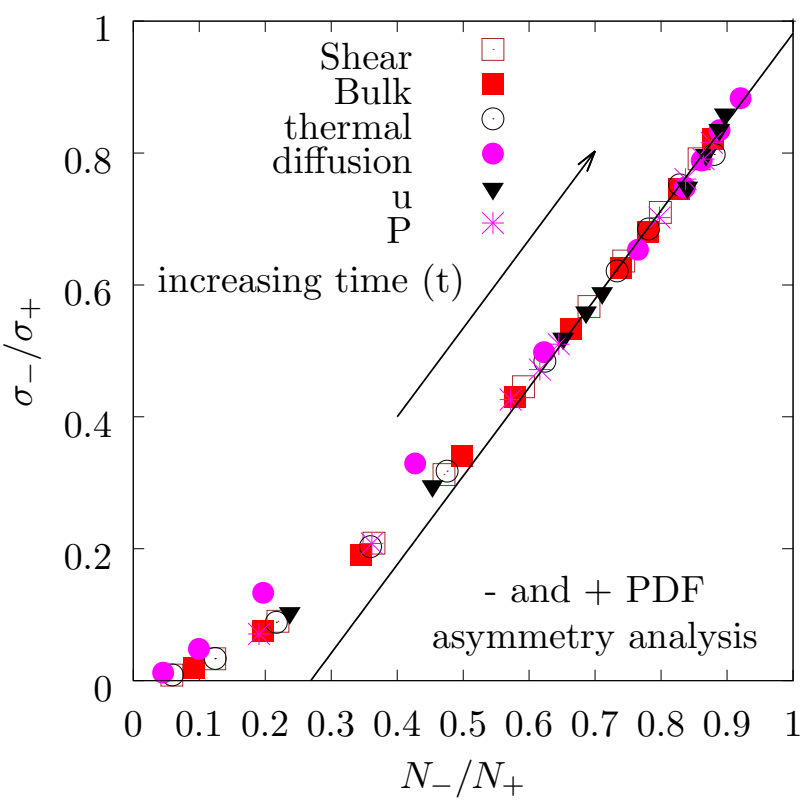

Figure 11 
(a)

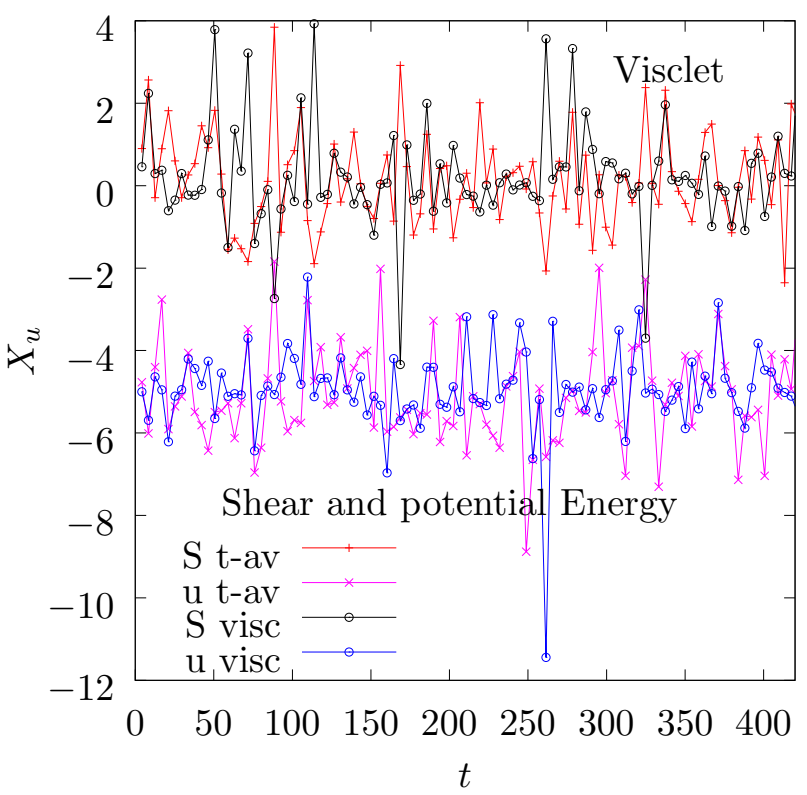

(b)

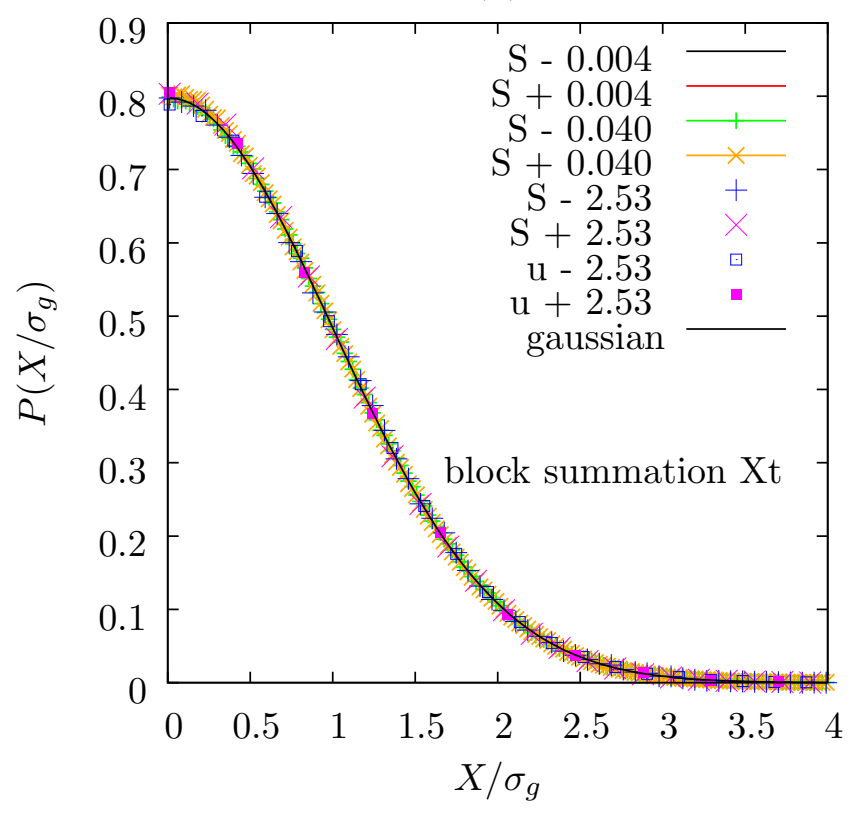

Figure 12 
(a)

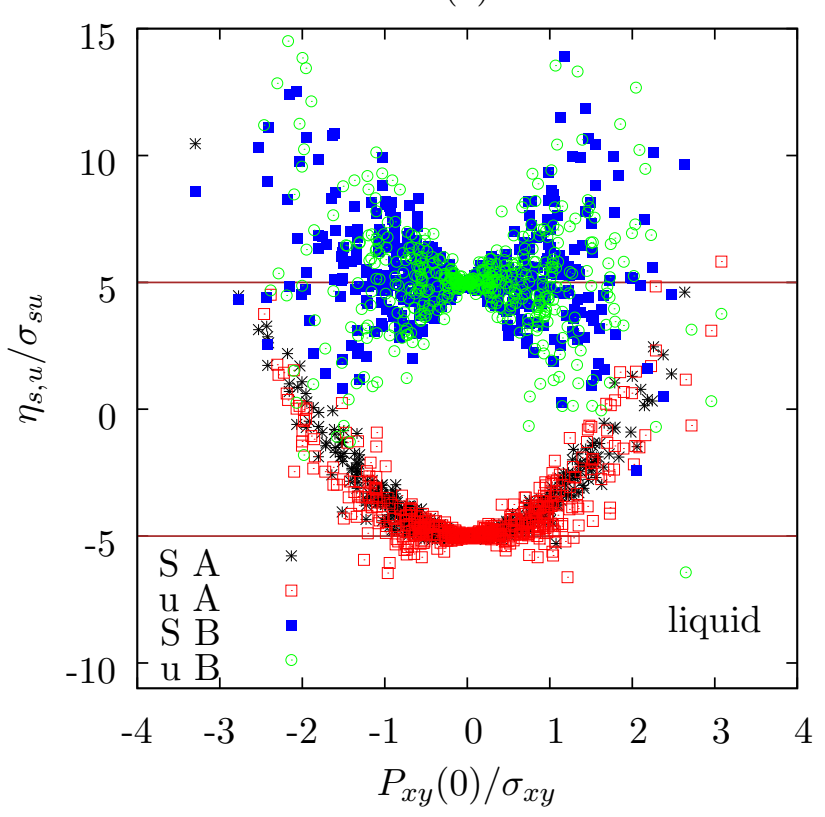

(b)

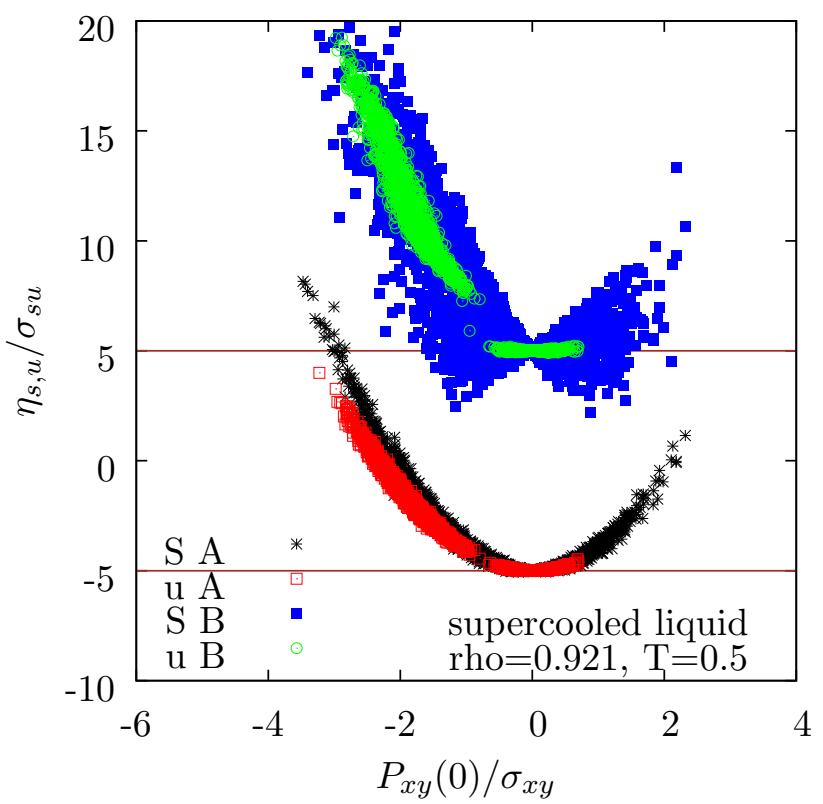

Figure 13 


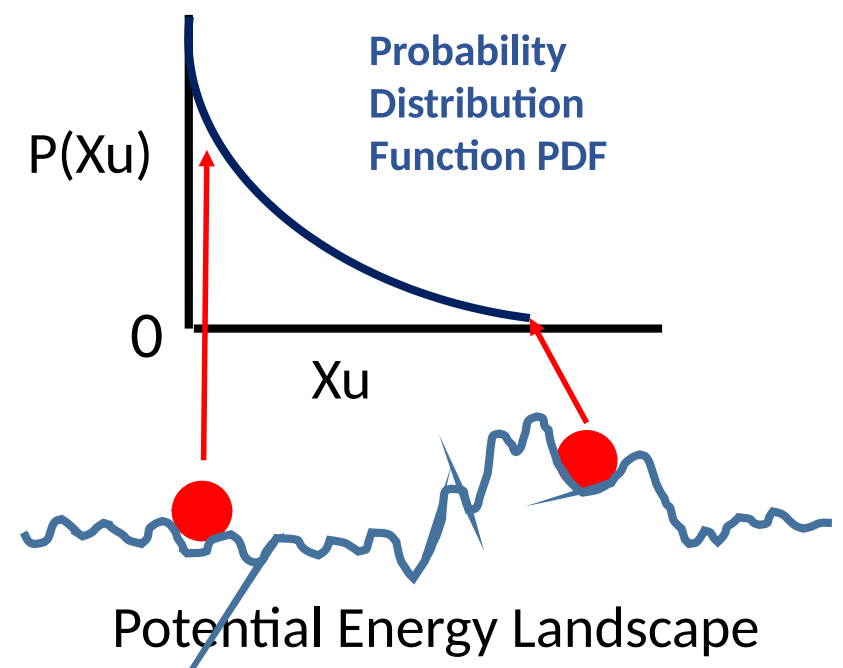

Figure 14 